\title{
SEGMENTACIÓN INTER E INTRARREGIONAL EN EL MERCADO LABORAL URBANO DE COLOMBIA \\ 2001-2006
}

\author{
Carlos Humberto Ortiz, José Ignacio Uribe y \\ Érika Raquel Badillo 1
}

\begin{abstract}
Resúmen
Utilizando la Encuesta Continua de Hogares para las trece áreas metropolitanas de Colombia, en este trabajo se examina la hipótesis de segmentación del mercado laboral urbano en cada año del período 2001-2006. Se encuentra evidencia de segmentación entre empresas formales grandes y el resto (segmentación intrarregional o de escala), y entre Bogotá y las demás áreas metropolitanas (segmentación interregional).
\end{abstract}

\begin{abstract}
Using the Continuous Household Survey for the thirteen metropolitan areas of Colombia, the hypothesis of segmentation of the urban labour market in Colombia is examined for each year of the period 2001-2006. Evidence of segmentation is found between large formal firms and the remainder (intra-regional or scale segmentation), and between Bogotá and other metropolitan areas (inter-regional segmentation).
\end{abstract}

Clasificación JEL: J21, J22, J24, J31, J41, J42.

\footnotetext{
${ }^{1}$ Ortiz y Uribe son profesores del Departamento de Economía de la Universidad del Valle, y Badillo es investigadora asociada. Se agradece la financiación y el apoyo de la Universidad del Valle. Este trabajo se realizó en el Grupo de Investigación en Economía Laboral. Correos electrónicos: ortizc@univalle.edu.co, josuribe@univalle.edu.co, erikabad84@hotmail.com .
} 


\section{Introducción}

¿Está segmentado el mercado laboral urbano de Colombia? ¿Incide la localización geográfica de las ciudades en la segmentación laboral? ¿Bastan los diferenciales de capital humano para explicar los diferenciales de salarios? Los analistas han tratado de contestar estas preguntas desde que existen encuestas de hogares en Colombia, pero los resultados han sido ambiguos.

Es usual que los analistas identifiquen agrupaciones del mercado laboral colombiano que presentan diferenciales salariales entre trabajadores comparables (Bourguignon, 1979; Magnac, 1991; Fields, 1980; Gracia, Hernández y Ramírez, 2001). Quienes analizan el efecto de la localización geográfica también observan que existen diferenciales salariales entre las áreas metropolitanas para trabajadores con las mismas características (Galvis, 2004; Romero, 2006). Sin embargo, cuando se está a punto de concluir que tales diferencias salariales entre agrupaciones y entre regiones implican una situación de segmentación laboral, los analistas relativizan sus conclusiones. Explican entonces que puede haber problemas de medición -vectores de precios y canastas de consumo diferentes-, o que existen factores inobservables o no incluidos que podrían explicar los diferenciales de remuneración -calidad de la educación, inteligencia, habilidad empresarial, gusto por la autonomía, etc.-, o que la segmentación es parcial, o una combinación de las anteriores. La respuesta típica a la pregunta sobre la existencia de segmentación laboral es sí, pero no...

Como la discusión no se ha resuelto empírica ni teóricamente, los hacedores de política económica han dictado sus reformas laborales como si los mercados laborales urbanos en Colombia estuvieran integrados, como si las políticas laborales operaran igual para las empresas grandes y para las pequeñas, como si los mercados laborales de Bogotá, Ibagué y Pasto fueran estructuralmente iguales o estuvieran integrados. En otras palabras, se ha supuesto que el mercado laboral urbano en Colombia es único.

Este estado de cosas no es satisfactorio. Es bien conocido que el tratamiento igual a los desiguales es inequitativo. Si los segmentos del mercado laboral se caracterizan por mecanismos de funcionamiento y ajuste diferentes, la propuesta de política no puede ser única, de otra forma se corre el riesgo de magnificar las diferencias.

Conviene entonces retomar las preguntas arriba planteadas. Se sugiere en este trabajo que un análisis de las remuneraciones laborales por tamaño de las empresas al tiempo que se controla por la dimensión regional pone más claramente en evidencia las relaciones de segmentación que se presentan entre empresas formales grandes, empresas formales pequeñas, micro y famiempresas, y trabajadores por cuenta propia sin calificación educativa tecnológica o profesional -esta clasificación de los trabajadores formales e informales se explicará posteriormente-.

Un esfuerzo anterior en este sentido utilizó información para las primeras 10 áreas metropolitanas de Colombia (Ortiz, Uribe y García, 2007). En este nuevo trabajo se amplía la gama regional utilizando la información de la Encuesta Continua de Hogares (ECH) para 
las 13 áreas metropolitanas. Adicionalmente, para tener en cuenta las diferencias de precios y canastas de consumo entre regiones, los ingresos de las diferentes áreas se deflactaron por los correspondientes índices locales de precios. También se mejoró la estimación del sesgo de selección de Heckman utilizando en la estimación de la participación laboral no sólo variables relacionadas con la condición socioeconómica sino también variables ficticias regionales. A diferencia del trabajo anteriormente mencionado, en este trabajo todas las regresiones corridas arrojaron evidencia significativa de sesgo de selección. No obstante, se puede comprobar fácilmente que el sesgo aunque significativo es pequeño y no modifica ni los signos ni la significación de las variables analizadas. De todas formas, todos los análisis se realizan sobre las regresiones corregidas.

Cabe advertir que este trabajo se centra sólo en las diferencias de remuneración laboral entre las áreas urbanas. Además, es generalmente reconocido que existen diferenciales de ingresos laborales significativos entre el sector rural y el urbano en Colombia, y se reconoce explícitamente la existencia de segmentación laboral rural-urbana (Leibovich, Nigrinis y Ramos, 2006).

Este documento consta de cinco partes. Después de esta introducción se realiza una revisión de literatura sobre los diferenciales salariales por regiones y por sectores en Colombia. Posteriormente, en la tercera sección, se explica la metodología de estimación utilizada. En la cuarta sección se presentan cinco modelos econométricos de estimación de la segmentación inter e intrarregional en las trece áreas metropolitanas de Colombia. Finalmente, en la sección quinta, se presentan las conclusiones.

\section{Estado del Arte}

\subsection{Segmentación Intrarregional}

Existe una amplia y reconocida literatura internacional sobre segmentación laboral. De hecho, la teoría del desarrollo económico ha planteado, desde hace más de medio siglo, que la caracterización más adecuada de los mercados laborales de los países subdesarrollados es la correspondiente al denominado dualismo: una coexistencia de sectores modernos y tradicionales, o tecnológicos y atrasados, o formales e informales (Lewis, 1954; Ranis y Fei, 1961; Fei y Ranis, 1964; Harris y Todaro, 1970; Doeringer y Piore, 1971; Stiglitz, 1974, 1982).

La proliferación de adjetivos para denominar a los subsectores que configuran una situación de dualismo económico es indicativa del grado de confusión que existe al respecto. De todas formas, la idea básica del dualismo es que existen algunos pocos sectores con suficiente acumulación de capital físico y humano que les permite aprovechar economías de escala y alcanzar niveles altos de productividad; por otro lado, se encuentra un conjunto importante de actividades económicas que se caracteriza por formas de producción precarias, intensivas en trabajo simple y de bajos niveles de productividad. A esta idea del dualismo se le asocia la de segmentación laboral, pues la permanencia de esta división estructural de la economía requiere que los trabajadores y los empresarios del sector 
informal encuentren barreras significativas para transitar hacia el sector formal, en el cual podrían encontrar mayor productividad y mayor ingreso. Atando cabos, no es difícil concluir que tales barreras se relacionan con restricciones de acceso a los mercados de capitales para invertir tanto en capital físico como humano (Uribe y Ortiz, 2006).

Desde la economía laboral también se ha planteado que los mercados laborales, especialmente los de los países subdesarrollados, se dividen en varios segmentos entre los cuales, por definición, la movilidad laboral es reducida o nula (OIT, 1972; Hart, 1973; Doeringer y Piore, 1975; Dickens y Lang, 1985; Taubman y Wachter, 1986; Solimano, 1988; Oroval y Escardíbul, 1998; Fields, 2004). En consecuencia, la restricción a la movilidad laboral implica la violación de la ley de un solo precio: el salario -precio del trabajo- varía dependiendo no sólo de las características intrínsecas del trabajador sino también de las características del sector donde el trabajador logra contratarse. Según Fields,

"The more modern labor market dualism literature stressed that for dualism to exist, different wages must be paid in different sectors to comparable workers” (Fields, 2004, p. 6).

En el campo de la economía laboral ha aparecido como contrapropuesta teórica que los mercados segmentados no son una caracterización precisa de la realidad, ni siquiera en los países subdesarrollados, y que el modelo tradicional neoclásico con un mercado integrado y competitivo puede representarlos adecuadamente. Para ello se plantea que las diferencias de remuneración laboral entre los sectores obedecen a factores no observables o no medibles como la calidad de la educación, la capacidad empresarial, la inteligencia, la disposición al trabajo, etc. (Cain, 1976; Rosenzweig, 1988; Maloney, 2003; Amaral y Quintín, 2003; BID, 2004).

Una de las visiones alternativas más fuertes en contra de la segmentación y el dualismo es la de la escogencia racional (De Soto, 1987; Maloney, 1998a, 1998b, 1999, 2003; BID, 2004). La idea en esta argumentación es que los informales no lo son por obligación sino por escogencia: ante la significativa injerencia estatal que incrementa los costos de la actividad económica -salarios mínimos, prestaciones laborales, tarifas incrementadas por formalización de la actividad económica, regulaciones de entrada -registro mercantil-, impuestos específicos a la actividad económica -impuesto de industria y comercio-, impuesto a las ventas -IVA-, pagos parafiscales, regulaciones higiénicas, regulaciones ambientales, costo de la corrupción oficial, etc.--, los empresarios deciden racionalmente abstenerse de formalizarse y permanecer en la informalidad. Desde esta perspectiva, la prevalencia de las actividades informales no es indicativa de precariedad sino de exceso de espíritu empresarial. Por ello, según esta visión, no existe segmentación (o dualismo), sino escogencia: los informales transitan hacia la formalidad cuando lo consideran conveniente dado el balance entre los beneficios y los costos de tal tránsito.

El BID, por ejemplo, critica la visión de los mercados segmentados, y en consecuencia el dualismo, argumentando que en algunos países subdesarrollados se observa cierta movilidad de los trabajadores entre los sectores formal e informal: el 16\% de la fuerza 
laboral mexicana y el $12 \%$ de la argentina entra o sale del sector informal en un semestre (BID, 2004, p. 76). Esto puede ser cierto,

"Nonetheless, the fact is that most workers remain in the sector in which they began” (Fields, 2004, p. 8).

Además, refiriéndose a la visión de Maloney, que hace énfasis en la escogencia de sector como explicación fundamental de la informalidad, el mismo Fields argumenta que

"(...) this model might fit the choice between formal sector employment and informal self-employment for those who already have the option of working in the formal sector, this is a limited group of people. Rather, (...), throughout the developing world, formal sector jobs appear to be far fewer in number than the number of people who want them. Thus, in my view, Maloney's view characterization applies to a subset of informal sector workers, but by no means all of them, nor probably event most” (Fields, 2004, p. 23).

Así, pues, Fields rescata la vieja idea estructuralista de la teoría del desarrollo económico sobre el dualismo como escasez de modernidad, como precariedad en el desarrollo de sectores tecnológicamente avanzados, sin descartar la posibilidad de que algunos trabajadores -aunque no la mayoría- escojan su condición de informales. Incluso el Banco Mundial, en una publicación reciente sobre los mercados laborales en América Latina, reconoce la coexistencia de informalidad laboral por escogencia (visión del “escape”), y de informalidad laboral por fuerza (visión de la “exclusión”) (Perry, Maloney, Árias, Fajnzylber, Mason, Saavedra-Chanduvi, 2007). De la misma forma, un análisis empírico reciente sobre tres países latinoamericanos (Argentina, México y Venezuela), y tres países en transición (Albania, Georgia y Ucrania), encuentra evidencia de segmentación entre los sectores, y diferenciales de movilidad por grupos: mientras los calificados -los que pueden escoger su condición- se mueven más fluidamente entre los sectores, la transición hacia el sector formal es mucho más difícil para los trabajadores por cuenta propia del sector informal -para los cuales la escogencia es menos factible-. En los países latinoamericanos se encuentra además evidencia de mejores salarios del sector formal, y una preferencia por pertenecer al mismo sector (Pagés y Stampini, 2007). Similarmente, Uribe y Ortiz (2006) sustentan una visión teórica que integra la informalidad como escape y como exclusión, según la tipología del Banco Mundial. Estos autores plantean que, si bien los costos que impone la institucionalidad sobre la actividad económica son significativos, ellos sólo son relevantes para un limitado grupo de empresarios y trabajadores que pueden escoger entre pagarlos o no; para la gran mayoría de la población laboral de los países subdesarrollados, sumidos en la pobreza, la adquisición de un registro mercantil sería algo tan exótico como pensar en adquirir licencia para pedir limosna. Simplemente esa posibilidad no está entre los rangos de escogencia porque la prioridad absoluta de los trabajadores pobres es la subsistencia. Para Uribe y Ortiz, la restricción a la movilidad laboral se explica por las restricciones de acceso tanto al capital físico como al capital humano. Los diferenciales de ingreso se explican, además, por la existencia de economías a escala en estos dos factores. También pueden ser importantes las diferencias de dotación infraestructural entre regiones. 
Estas diferencias se podrían medir en la existencia y competencia de las instituciones públicas, la cantidad y calidad de la dotación física regional: servicios públicos, carreteras y vías, otros medios de comunicación, centros educativos, cámaras de comercio, etc. Por ello, el grado de modernidad de las regiones -usualmente medido con variables sustitutas o con el grado de industrialización- incide en su grado de informalidad (Uribe y Ortiz, 2006; García, 2005).

\subsection{Segmentación Interregional}

Además de la segmentación intrarregional, lo cual implica restricciones a la movilidad entre empresas de una misma región, también es factible que existan restricciones a la movilidad interregional de los trabajadores.

Utilizando datos actualizados sobre las cuentas económicas departamentales (DANE, varios años; y CEGA, 2006), los analistas del desarrollo económico han acumulado una evidencia empírica contundente sobre la divergencia del ingreso per cápita entre los departamentos de Colombia (Bonet y Meisel, 2007, 1999; Barón, 2004; Birchenall y Murcia, 1996; Meisel, 1993; Mora y Salazar, 1994; Rocha y Vivas, 1998). Estos análisis han revelado que la brecha de ingresos entre Bogotá y el resto del país, se ha venido ampliando; también han revelado que la clasificación ordinal de los departamentos según nivel de ingreso per cápita tiende a mantenerse en el tiempo, en especial los departamentos pobres tienden a mantener su condición.

De paso, este comportamiento regional en Colombia es similar al proceso de divergencia internacional del ingreso per cápita tal como lo han documentado Maddison (1994), Pritchett (1997), Hall y Jones (1997), Easterly y Levine (2001), y Perry, Arias, Maloney, López y Servén (2006). En estos trabajos también se encuentra que la pobreza es persistente y que los países más desarrollados tienden a crecer más rápidamente en el largo plazo.

Es posible concluir de los anteriores análisis que las diferencias entre las regiones y entre las naciones juegan un papel importante en la determinación de la remuneración de los factores. Esa divergencia interregional es consistente con la existencia de barreras a la movilidad de los factores entre las regiones. Debe ser así porque de otra forma la movilidad factorial -tanto del capital como del trabajo- tendería a igualar las remuneraciones factoriales entre regiones. Por supuesto, es posible verificar que a pesar de la existencia de procesos migratorios de las regiones pobres a las ricas, los diferenciales de ingreso per cápita se mantienen en el tiempo. Un trabajo reciente sobre la productividad del sector manufacturero también estima diferenciales significativos entre las áreas metropolitanas de Colombia (Iregui, Melo y Ramírez, 2007).

Ya en el campo específico de la economía laboral, Galvis (2004) analiza la integración regional. Este estudio utiliza la información trimestral de la Encuesta Nacional de Hogares (ENH) en el periodo 1984-2000 para las áreas metropolitanas de Bogotá, Barranquilla, Manizales, Bucaramanga y Pasto. Con la información local se construyen las 
correspondientes series de salario real promedio por hora. La definición de integración del mercado laboral que se adopta es la de convergencia en los salarios. Sus resultados muestran que existe una integración parcial del mercado laboral: existe integración para la fracción de la población con niveles educativos primarios y secundarios, pero no para la población con nivel educativo superior. Galvis argumenta, sin demostración, de que si se tuvieran en cuenta otras variables relevantes, como los costos de transporte y los costos de vida por región, los resultados obtenidos podrían ser más contundentes a favor de la integración regional del mercado laboral colombiano.

Romero (2006) por su parte analiza las diferencias regionales de los salarios utilizando la información de la Encuesta Continua de Hogares (ECH) de enero de 2001 a junio de 2004. Se analizan doce de las trece principales áreas metropolitanas de Colombia: Bogotá, Cali, Medellín, Barranquilla, Bucaramanga, Manizales, Pasto, Pereira, Cúcuta, Montería, Cartagena y Villavicencio. El trabajo comprende la estimación de cuatro modelos: el primero es una ecuación de ingresos donde sólo se incorporan como determinantes los efectos regionales. En el segundo modelo se amplía el conjunto de regresores y se incorporan cinco variables ficticias de nivel educativo. En el tercer modelo se incluyen otras características personales (edad, estratificación de la vivienda, género, posición del individuo en el hogar), variables económicas (tipo de ocupación, rama de actividad económica), y variables laborales (seguridad social en salud, pensión, contrato laboral escrito y empleo de tiempo parcial). El cuarto y último modelo incluye las variables del tercer modelo, pero descuenta del ingreso laboral el efecto del costo de vida por ciudad. Los resultados del primer modelo muestran grandes diferencias en el ingreso laboral. Entre Bogotá y Montería se estima una diferencia del 55\%. Se comprueba que las ciudades de mayor tamaño tienen mayores ingresos laborales. Los resultados del segundo modelo muestran que existe una diferencia en remuneración de aproximadamente el $40 \%$ entre los ocupados localizados en Medellín con respecto a los ubicados en Pasto, Montería y Barranquilla. Cuando se controla por nivel educativo la diferencia se ubica por debajo del 20\%. El efecto tamaño de las ciudades en los diferenciales salariales se mantiene en este modelo. En el tercer modelo se controla por educación, características del individuo, tipo de empleo y actividad económica. Como resultado se identifican tres “clubes” de convergencia: 1) el primer “club” está compuesto por Bogotá, Medellín y Cali, ciudades en las cuales los ocupados reciben en promedio un ingreso laboral entre 10 y 15\% más alto que el promedio de ingreso de las doce ciudades analizadas, 2) en el segundo "club" se ubican Bucaramanga, Villavicencio, Cartagena, Pereira y Cúcuta, y 3) en el tercer "club” están Manizales, Pasto, Montería y Barranquilla. El cuarto modelo utiliza como variable dependiente el ingreso laboral de las ciudades deflactado por los índices de precios locales. Los resultados de este modelo muestran que las diferencias regionales llegan a un máximo de 22\% para Barranquilla, mientras que para las otras ciudades las diferencias son mínimas y no significativas. En síntesis, el estudio de Romero (2006) muestra que la hipótesis de segmentación laboral no es muy fuerte. Se argumenta que las diferencias en los ingresos laborales entre ciudades pueden deberse a una combinación de factores no examinados en el estudio: diferencias regionales en la participación laboral, diferencias en el tipo y condiciones de empleo, diferencias en la actividad económica predominante, y diferencias en las dotaciones de capital humano. Lo anterior implica que el mercado laboral de 
Colombia no tiene discrepancias regionales, aunque se presenta algún grado mínimo de segmentación regional no siempre significativo. Sin embargo, el sistemático menor nivel de ingresos laborales de Barranquilla es un argumento a favor de la hipótesis de segmentación regional.

\section{Hipótesis y Metodología}

Este trabajo plantea como hipótesis que la segmentación laboral colombiana tiene dos dimensiones: una sectorial, que obedece fundamentalmente a las restricciones de acceso al capital físico y al capital humano, y que limita la movilidad hacia las empresas de mayor tamaño en una misma región; y otra regional, que obedece fundamentalmente a las barreras de movilidad del trabajo y de otros factores entre regiones. A estas dos dimensiones las denominamos segmentación intrarregional (o de escala), y segmentación interregional.

Bajo la hipótesis de que existen economías a escala en las empresas, y de que también existen fuertes restricciones de acceso al capital físico y al capital humano, en este trabajo se postula que existe mayor movilidad laboral entre empresas del mismo tamaño, y menor movilidad entre empresas de diferente tamaño, especialmente desde las más pequeñas hacia las mayores. La hipótesis de economías a escala o de tamaño implica que las mayores empresas disfrutan de mayor productividad y, en consecuencia, realizan mayores ganancias y gozan de mayores remuneraciones (Uribe y Ortiz, 2006).

Teniendo en cuenta esta hipótesis y también la disponibilidad de información de las encuestas de hogares, las empresas se clasifican de acuerdo con los siguientes tamaños de planta:

1) Unipersonal: este grupo está conformado fundamentalmente por trabajadores por cuenta propia no profesionales ni técnicos, y por trabajadores domésticos. Se caracterizan por trabajar solos y tener una escasa formación educativa.

2) Famiempresa: negocio con una planta entre 2 y 5 trabajadores.

3) Microempresa: negocio con una planta entre 6 y 10 trabajadores.

En la clasificación del DANE se consideran informales las siguientes categorías ocupacionales: trabajadores familiares sin remuneración, trabajadores domésticos, trabajadores por cuenta propia no profesionales ni técnicos, y trabajadores y patrones en empresas de hasta diez trabajadores. Por consiguiente, los primeros tres tipos de empresas arriba definidos simplemente reorganizan a los informales según el tamaño de planta. Los siguientes dos tipos de empresas pertenecen al sector formal:

4) Formal Pequeña: empresa de hasta 10 trabajadores que incluye profesionales o técnicos (la mayoría está conformada por profesionales o técnicos por cuenta propia). 
5) Formal Grande: empresa con más de 10 trabajadores.

Es importante tener en cuenta que la Encuesta Continua de Hogares (ECH) del DANE le pregunta al trabajador por el número de trabajadores de la empresa en la cual labora. Así, pues, en este trabajo se utiliza el número de trabajadores como medida del tamaño de planta. Hubiera sido conveniente usar otras medidas del tamaño de planta de las empresas producción, ventas o activos-, pero esta información no está disponible en las encuestas de hogares.

Una primera aproximación a la hipótesis del tamaño de planta (las remuneraciones tienden a aumentar con el tamaño de planta de las empresas) se muestra en la Grafica 1.

En el eje de las abscisas se ordenan las trece áreas metropolitanas de Colombia según la remuneración media del sector formal grande. En el eje de las ordenadas se mide el ingreso medio a precios constantes de 1998 teniendo en cuenta el índice de precios al consumidor de cada una de las áreas incluidas. Para cada ciudad se estima el ingreso medio de los subsectores según tamaño de planta.

Gráfica 1

Ingreso Medio por Tamaño de Planta y Área Metropolitana 2006

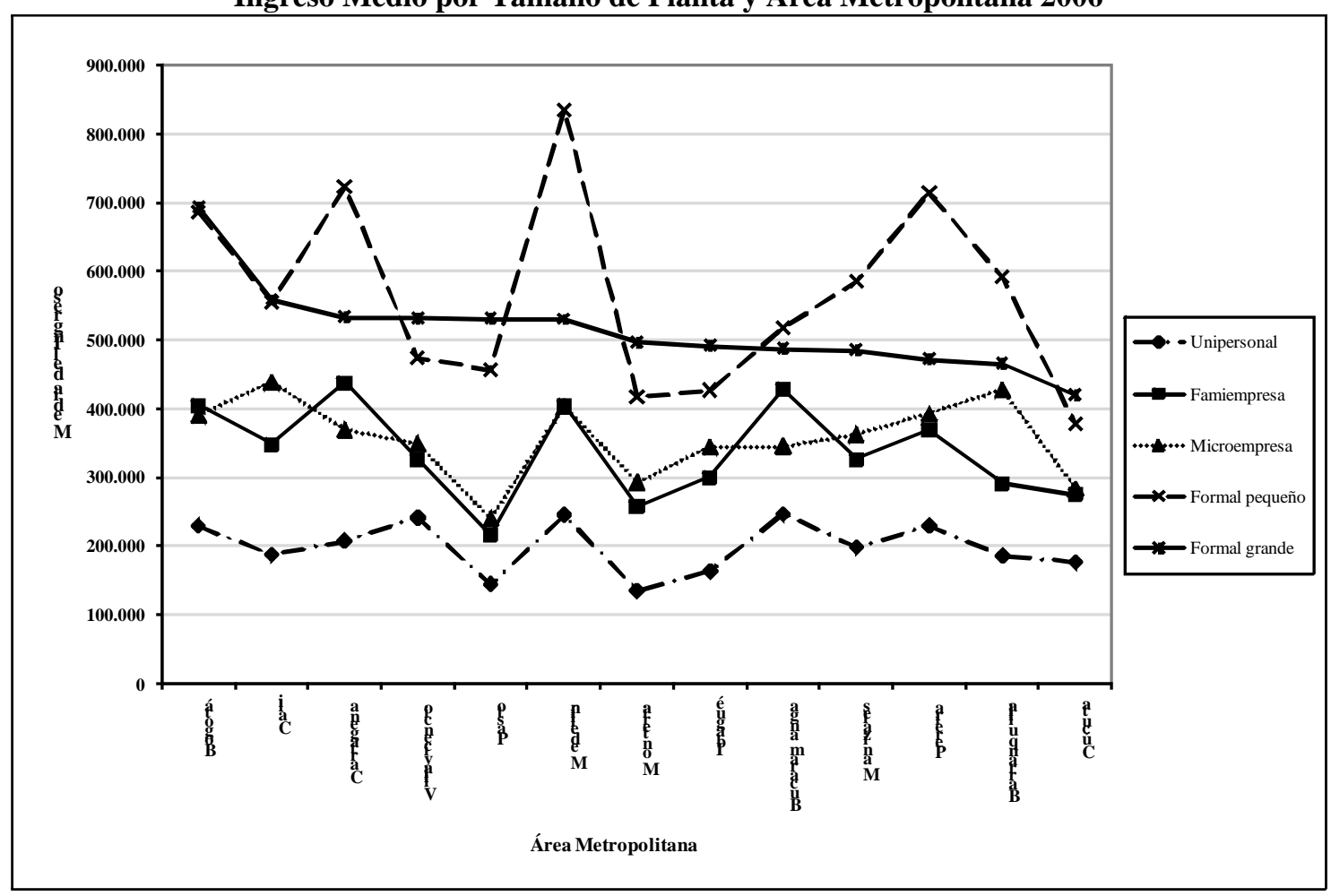

Fuente: Procesamiento propio a partir de ECH (DANE).

Con base en la Gráfica 1 se obtienen varias conclusiones: 
1. Existe una gran dispersión de los ingresos: el sector formal (compuesto por los subsectores formal pequeño y formal grande) tiene sistemáticamente mayores remuneraciones salariales que el sector informal (compuesto por los subsectores unipersonal, famiempresa y microempresa).

2. Aunque existen diferencias en la remuneración media de los formales pequeños y grandes en algunas áreas, como en el caso de Medellín y Pereira, este no es un fenómeno sistemático, y más bien se encuentra una gran coincidencia de los niveles de remuneración laboral media de estos subgrupos, como en los casos de Bogotá y Cali. Sin embargo, esta igualación de las remuneraciones medias no debe inducir a error: si se tiene en cuenta que el trabajador típico del sector formal pequeño es un técnico o profesional independiente que cuenta en 2006 con 17.5 años de educación, mientras que el trabajador promedio del sector formal grande sólo tiene en promedio 13 años de educación, es evidente que por cada año de educación de los últimos se recibe una mayor remuneración -las remuneraciones marginales difieren-. De hecho, el análisis econométrico también revelará la existencia de segmentación laboral entre los formales grandes y los pequeños.

3. Los ingresos medios de los trabajadores en fami y microempresas son prácticamente iguales en cada ciudad.

4. La menor remuneración media en todas las áreas metropolitanas le corresponde a los unipersonales.

5. Por último, pero no por eso menos importante, la hipótesis de que la remuneración laboral aumenta con el tamaño se sostiene. Para entenderla se debe considerar que el sector formal pequeño puede serlo por tamaño de planta, pero no por acumulación de capital humano: como se mencionó anteriormente, los profesionales y técnico independientes cuentan con un acervo educativo muy superior al del resto de sus colegas trabajadores. Además, el capital físico del sector formal pequeño probablemente es proporcional a su dotación de capital humano, pues está constituida por equipo intensivo en tecnología (piénsese por ejemplo en el equipo requerido por un odontólogo). Cabe resaltar que la hipótesis del efecto tamaño se observa más claramente cuando se comparan los tamaños por área metropolitana; la remuneración media según tamaño, R, se ordena de la misma forma en todas las áreas metropolitanas:

$$
\mathrm{R}(\text { Unipersonal })<\mathrm{R}(\text { Famiempresa }) \approx \mathrm{R}(\text { Microempresa })<\mathrm{R}(\text { Formal Pequeña }) \approx \mathrm{R}(\text { Formal Grande }) \text {. }
$$

En cambio, la comparación por tamaño sin tener en cuenta el efecto regional puede llevar a conclusiones equivocadas. Por ejemplo, como muestra la Gráfica 1, la remuneración media del sector formal grande en Cúcuta o en Barranquilla es similar a la remuneración media de las famiempresas en Bucaramanga, Medellín, Cartagena y Bogotá.

Para la estimación de las ecuaciones de ingreso se utilizaron errores estándar robustos a la heterocedasticidad del tipo White. Es común en la estimación de estos modelos utilizar la técnica de Heckman (1979) para la corrección del sesgo de selectividad. Se trabajó con la 
versión de la metodología de Heckman en la que se estima de manera simultánea un modelo Probit de participación laboral y la ecuación de ingresos por el método de máxima verosimilitud. En el modelo Probit se calcula la probabilidad que tiene cada individuo de participar en el mercado laboral y se incorpora como regresor (el lambda de Heckman) en la ecuación de ingresos. En la ecuación de participación laboral se incluyeron variables explicativas relacionadas con la oferta laboral y el costo de oportunidad de no trabajar. Como indicadores de la oferta laboral se incluyeron los años aprobados de educación y la experiencia potencial, y variables de características socioeconómicas: género y la condición de jefe de hogar. Como indicadores del costo de oportunidad de no trabajar se incluyó la tasa de desempleo del hogar, definida como el número de desempleados en el hogar dividido por el número de participantes. Se incluyeron dos variables que reflejan el impacto de la dependencia infantil y juvenil: el número de hijos en el hogar de 0 a 6 años, y el número de hijos entre los 7 y 18 años. Finalmente, se incluyeron variables ficticias de las áreas metropolitas para capturar el efecto regional sobre la participación laboral.

\section{Estimaciones Econométricas}

\subsection{Primer Modelo: Regresión Minceriana}

La propuesta metodológica de este trabajo se basa en la tradición analítica de la teoría del capital humano. Se postula que los ingresos laborales se explican por factores relacionados primordialmente con la oferta laboral: la acumulación de capital humano (educación y experiencia) y las características socioeconómicas (género y jefatura del hogar). Se supone que el capital humano aumenta la productividad pero de forma decreciente; esto último es particularmente relevante con respecto a la experiencia o el aprendizaje en la práctica. La determinación de las remuneraciones también es definida por las características socioeconómicas de los individuos. Por el lado de la oferta laboral, los hombres parecen enfrentar una mayor presión social por conseguir remuneraciones mayores; además, por el lado de la demanda laboral, la existencia de discriminación laboral femenina puede incidir en una mayor remuneración masculina. Se espera encontrar, por tanto, una relación positiva entre la condición masculina y los ingresos. Por otra parte, la condición de jefe de hogar también incide en la participación y en la generación de ciertos mínimos de ingreso para enfrentar el problema de la subsistencia. Se espera, así, que la jefatura del hogar se relacione positivamente con la remuneración laboral. La especificación funcional de estas consideraciones se cristaliza en la regresión de Mincer, cuya forma típica es la siguiente:

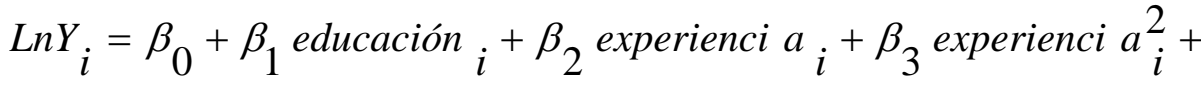

$$
\begin{aligned}
& \beta_{4} \text { género }_{i}+\beta_{5} \text { jefe }_{i}+e_{i} \text {, }
\end{aligned}
$$

Donde $\operatorname{LnY}$ es el logaritmo del salario real por hora, Educación es el número de años aprobados de educación formal, Experiencia es la experiencia potencial (= edad - años de educación - 5), Género es la variable binaria para el género masculino (1 para hombres y 0 para mujeres), Jefe es la variable binaria de la jefatura del hogar (1 para jefes, 0 de otra manera), y e es la perturbación aleatoria. 
Las regresiones Mincerianas se muestran en el Cuadro 1. Los coeficientes tienen los signos esperados: el ingreso aumenta con la educación; igual sucede con la experiencia; la experiencia al cuadrado se relaciona negativamente con el ingreso -la rentabilidad de la experiencia es decreciente-; el género masculino se relaciona positivamente con el ingreso; y la jefatura del hogar también. Las estimaciones son siempre significativas al 1\% con excepción de la variable género que no es significativa en 2001-2002.

Cuadro 1

Ecuaciones Mincerianas Simples

\begin{tabular}{|c|c|c|c|c|c|c|c|c|c|c|c|c|c|}
\hline & & 2001 & & 2002 & & 2003 & & 2004 & & 2005 & & 2006 & \\
\hline Sin & constante & $-5,9832$ & *** & $-6,0439$ & *** & $-6,0039$ & *** & $-6,0443$ & $* * *$ & $-6,0420$ & *** & $-5,9136$ & *** \\
\hline $\mathrm{C}$ & educación & 0,1145 & 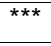 & 0,1149 & 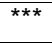 & 0,1103 & *** & 0,1104 & 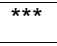 & 0,1119 & $\star \star \star$ & 0,1061 & $\star \star \star \star$ \\
\hline $\mathrm{O}$ & experiencia & 0,0253 & *** & 0,0277 & 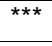 & 0,0242 & 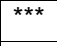 & 0,0278 & 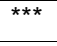 & 0,0261 & 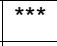 & 0,0239 & $\star \star \star \star$ \\
\hline $\mathrm{R}$ & experiencia2 & $-0,0003$ & *** & $-0,0003$ & $\star \star \star$ & $-0,0003$ & $\star \star \star \star$ & $-0,0003$ & $\star \star * *$ & $-0,0003$ & *** & $-0,0002$ & 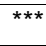 \\
\hline $\mathrm{R}$ & género & 0,0206 & ** & 0,0206 & ** & 0,0626 & 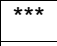 & 0,0665 & 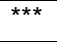 & 0,0973 & 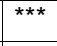 & 0,0840 & $\star \star \star$ \\
\hline $\mathrm{E}$ & jefe & 0,1521 & 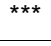 & 0,1517 & 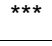 & 0,1530 & 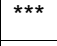 & 0,1615 & 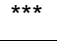 & 0,1536 & 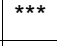 & 0,1644 & 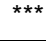 \\
\hline $\mathrm{C}$ & & & & & & & & & & & & & \\
\hline $\mathrm{C}$ & $\mathrm{R} 2$ & 0,3393 & & 0,3500 & & 0,3521 & & 0,3475 & & 0,3622 & & 0,3502 & \\
\hline I & Akaike & 80341,2 & & 76270,6 & & 75748,2 & & 76617,3 & & 83217,0 & & 81937,1 & \\
\hline Ó & Schwarz & 80392,1 & & 76321,2 & & 75799,0 & & 76668,2 & & 83268,5 & & 81988,6 & \\
\hline $\mathrm{N}$ & $\mathrm{N}$ & 36114 & & 34479 & & 35000 & & 35502 & & 39458 & & 39982 & \\
\hline $\mathrm{C}$ & constante & $-5,8848$ & 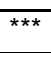 & $-5,8811$ & 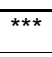 & $-5,8664$ & 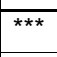 & $-5,8517$ & 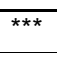 & $-5,9645$ & 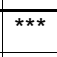 & $-5,7249$ & 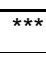 \\
\hline $\mathrm{O}$ & educación & 0,1133 & $* \star *$ & 0,1128 & $* \star *$ & 0,1086 & *** & 0,1080 & $\star \star \star *$ & 0,11099 & *** & 0,1036 & $\star \star \star$ \\
\hline $\mathrm{R}$ & experiencia & 0,0220 & 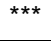 & 0,0221 & 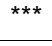 & 0,0195 & 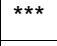 & 0,0213 & 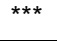 & 0,02344 & *** & 0,0175 & $\star \star \star$ \\
\hline $\mathrm{R}$ & experiencia2 & $-0,0002$ & $\star * \star$ & $-0,0002$ & $* \star \star$ & $-0,0002$ & 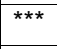 & $-0,0002$ & 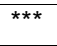 & $-0,0002$ & $* * *$ & $-0,0001$ & $\star \star \star \star$ \\
\hline $\mathrm{E}$ & género & 0,0076 & & $-0,0001$ & & 0,0442 & 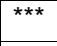 & 0,0395 & 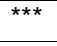 & 0,0853 & 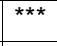 & 0,0548 & $\star \star \star$ \\
\hline $\mathrm{C}$ & jefe & 0,1298 & *** & 0,1172 & *** & 0,1244 & *** & 0,1225 & *** & 0,1385 & *** & 0,1282 & 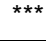 \\
\hline $\mathrm{C}$ & & & & & & & & & & & & & \\
\hline I & lambda & $-0,0635$ & *** & $-0,1017$ & *** & $-0,0866$ & *** & $-0,1202$ & 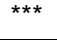 & $-0,0502$ & *** & $-0,1222$ & 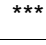 \\
\hline Ó & & & & & & & & & & & & & \\
\hline $\mathrm{N}$ & $\mathrm{N}$ & 36114 & & 34479 & & 35000 & & 35502 & & 39458 & & 39982 & \\
\hline \multicolumn{14}{|c|}{${ }^{*} \mathrm{p}<.1 ;{ }^{* *} \mathrm{p}<.05 ;$ *** $\mathrm{p}<.01$} \\
\hline
\end{tabular}

Fuente: Procesamiento de la ECH 2001-2006 II Trimestre. Cálculos de los autores.

\subsection{Segundo Modelo: Regresión Minceriana con Variables de Tamaño por Rangos}

Los estudios de rentabilidad de la educación que utilizan las ecuaciones de Mincer se basan en variables del lado de la oferta, como las regresiones mincerianas sencillas que se acaban de presentar. Esta especificación sería adecuada si el mercado laboral fuera único, integrado y competitivo, como supone usualmente la teoría del capital humano. Pero ninguna de las condiciones mencionadas está garantizada. De hecho, una larga tradición teórica ha considerado conveniente suponer que existen estructuras económicas segmentadas para entender el comportamiento de las economías, especialmente el de las economías subdesarrolladas (Lewis, 1954; Doeringer y Piore, 1971 y 1975; Piore, 1975, 1980 y 1983; Taubman y Wachter, 1986; Saint-Paul, 1996; Lang y Dickens, 1984, 1985 y 1987; Solimano, 1988). Y también se verifica que los mercados laborales se caracterizan por evidentes imperfecciones: existencia de sindicatos y otros mercados internos de trabajo, fijación de salarios de eficiencia, existencia de señalización y credencialismo, etc. 
En la tradición teórica de la segmentación se supone usualmente que existen dos mercados laborales relativamente independientes: uno moderno o formal, que se caracteriza por "buenos" empleos, y otro atrasado o informal, el cual ofrece empleos "malos". Los puestos "buenos" son aquellos que cumplen determinadas características: estabilidad y seguridad, elevados y crecientes salarios, escalas de ocupación, utilización de tecnologías relativamente avanzadas e intensivas en capital, presencia de una gestión eficiente y, en muchos casos, la existencia de sindicato. Además, existe un racionamiento de estos "buenos" puestos de trabajo. De otro lado, los mercados de trabajo del sector informal transan puestos de trabajo "malos" y tienen características opuestas a los del sector primario (McConnell y Brue, 1997; Doeringer y Piore, 1975). La persistencia de estas diferencias se explica por la existencia de fuertes barreras a la movilidad laboral entre los sectores mencionados.

Para contrastar la hipótesis de segmentación desde el punto de vista empírico se tendría que encontrar que las diferencias de ingresos entre los sectores no responden sólo a diferencias en capital humano. Es decir, se debe comprobar que las remuneraciones laborales también se asocian con la pertenencia a un determinado sector. Para realizar esta comprobación se estiman ecuaciones de Mincer utilizando variables ficticias sectoriales; si los coeficientes asociados con las variables ficticias son significativos, al tiempo que se controla por las variables de capital humano (educación y experiencia), se puede concluir que el diferencial de remuneraciones es atribuible a factores sectoriales. Ello implicaría, además, barreras a la movilidad del trabajo, pues sólo de esta forma se podría explicar que un profesional, por ejemplo, con un nivel de capital humano dado acepte una menor remuneración en un sector que en otro (Bourguignon, 1979, 1983; Fields, 1980; Magnac, 1991; Lang y Dickens, 1984; Maloney, 1998a, 1998b).

Este tipo de análisis tiene por supuesto limitaciones, pues algunas variables relacionadas con la productividad no necesariamente son observables o medibles (habilidad del trabajador, inteligencia, preferencia por la independencia, calidad de la educación, ambiente familiar, capital social, etc.). Este recurso ha sido muy útil para descalificar los análisis que encuentran alguna evidencia de segmentación (Bourguignon, 1979; Fields, 1980; Maloney, 1998a, 1998b). No obstante, en este trabajo seguimos el procedimiento usual porque la imposibilidad de capturar algunas variables relevantes no necesariamente anula las evidencias de segmentación que las variables sectoriales arrojan, especialmente si no se conoce qué tan relevantes son las variables excluidas. Por otra parte, la evidencia de segmentación que se obtiene con el procedimiento usual debe ser seriamente considerada si las variables que se incluyen tienen un soporte teórico y, además, la evidencia de segmentación es temporalmente consistente.

La introducción de variables sectoriales en el análisis de segmentación se ha llevado a cabo de varias formas: clasificando a los trabajadores por sectores económicos o por nivel de ingreso. Siguiendo la propuesta estructuralista que se plantea en Uribe y Ortiz (2006), en este trabajo se supone que los ingresos dependen del tamaño de la empresa. Un modelo de equilibrio económico general que genera segmentación laboral de escala se encuentra en 
Ortiz y Uribe (2004b). Este enfoque implica entonces abandonar el supuesto simple de que sólo la oferta laboral determina las remuneraciones.

La hipótesis central del trabajo de Uribe y Ortiz (2006) es que la tecnología de las empresas se caracteriza por rendimientos crecientes a escala tanto en capital humano como en capital físico. Además, se postula que existen fuertes complementariedades productivas entre estas formas de capital (Hirschman, 1958; Griliches, 1969). Ambos supuestos implican que los ingresos de las empresas y de sus trabajadores aumentan con el tamaño de planta (Reilly, 1995).

La mayor remuneración de los trabajadores en las empresas grandes también se puede explicar por el mayor poder de mercado de éstas. La generación de rentas asociada al poder de mercado les permite a los trabajadores asociados en sindicatos negociar mejores remuneraciones (Mellow, 1982). Otra explicación microeconómica de los mayores salarios se encuentra en la teoría de los salarios de eficiencia: Rebitzer y Robinson (1991), y SaintPaul (1996) argumentan que las mayores remuneraciones en las empresas grandes responden a la mayor dificultad de monitoreo y control del trabajo, lo cual obliga a las empresas a ofrecer mayores salarios para inducir el esfuerzo y la dedicación. Para explicar los mayores ingresos de las empresas grandes también es posible postular que éstas se benefician de externalidades productivas de las cuales no se pueden beneficiar las empresas pequeñas. Algunos argumentos señalados por la teoría económica para sustentar esta última visión son los siguientes: 1) las empresas pequeñas pueden sufrir restricciones crediticias por asimetrías de información y por su incapacidad de garantizar los préstamos con garantías reales (colateral); 2) las empresas pequeñas pueden no tener acceso a ciertos servicios públicos en razón de su informalidad; entre estos servicios se encuentran la protección a los derechos de propiedad, el apoyo legal para el cumplimiento de contratos, la disponibilidad de información sobre oportunidades económicas que se canalizan a través de fuentes oficiales (De Soto, 1987; Loayza, 1997).

La hipótesis de segmentación por tamaño de planta implica que existen fuertes restricciones para acceder al mercado de capitales, de tal manera que la inversión en capital físico y en capital humano está restringida. Por tanto, la movilidad laboral de una empresa pequeña a una grande está también relativamente restringida por la dificultad de acumular los mínimos requeridos de capital físico y capital humano. En consecuencia, los trabajadores encuentran escasas posibilidades de entrar a competir en las empresas con mayor acumulación, y se deben conformar con permanecer en las empresas pequeñas, trabajar con tecnología subóptima, y devengar menores remuneraciones.

Uno de los patrones típicos de los mercados de trabajo es que la remuneración de trabajadores iguales tiende a aumentar con el tamaño de la firma. Brown y Medoff (1989) identifican un diferencial significativo de ingresos entre empresas grandes y pequeñas de los Estados Unidos. Albæk et al (1998) encuentran resultados similares para los países nórdicos de Europa. Brunello y Colussi (1998) también encuentran evidencia significativa de la relación salario-tamaño para Italia. Main y Reilly (1993) presentan evidencia significativa de esta relación para el caso de Gran Bretaña. Oosterbek y Van Praag (1995) 
la reportan para el caso de Holanda. También se encuentra evidencia significativa para países hispanoamericanos. Huguet y Sánchez (2001) muestran la relación remuneracióntamaño para el caso español. Perlbach y Calderón (1998) muestran evidencia de que las remuneraciones salariales en Mendoza (Argentina) se relacionan positiva y significativamente con el tamaño de la planta.

Para contrastar la anterior hipótesis se introducen variables ficticias según los tamaños de planta que se definieron anteriormente: unipersonal, famiempresa, microempresa y formal pequeño. Se excluye el grupo formal grande porque se toma como referencia. Esta clasificación sólo se puede realizar cuando la encuesta de hogares incluye el módulo de informalidad (junio). Ante la imposibilidad de medir directamente el tamaño de las empresas, se incluyen en las regresiones mincerianas las variables binarias de tamaño por rango. El supuesto heroico de este procedimiento es que el mayor tamaño de planta implica una mayor disponibilidad de capital físico y de capital humano. Es éste, obviamente, un supuesto burdo, pero es el único posible dadas las restricciones que plantea la información disponible. Así, la especificación de las regresiones mincerianas modificada por las variables de escala, es la siguiente:

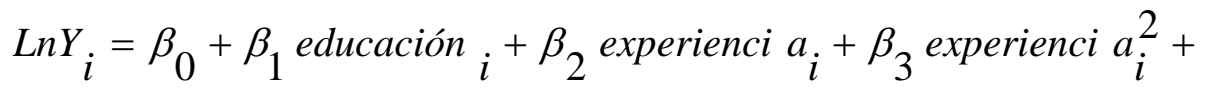

$$
\begin{aligned}
& \beta_{4} \text { género }_{i}+\beta_{5} \text { jefe }_{i}+\beta_{6} \text { unipersona } l_{i}+\beta_{7} \text { famiempres } a_{i}+ \\
& \beta_{8} \text { microempre sa }_{i}+\beta_{9} \text { formal }_{\text {pequeña }}+e_{i}
\end{aligned}
$$

Los resultados de estas regresiones junto con la correspondiente corrección por el sesgo de selección se presentan en el Cuadro 2.

Cuadro 2

\begin{tabular}{|c|c|c|c|c|c|c|c|c|c|c|c|c|c|}
\hline & & 2001 & & 2002 & & 2003 & & 2004 & & 2005 & & 2006 & \\
\hline \multirow{15}{*}{$\begin{array}{c}\text { Sin } \\
\text { C } \\
\text { O } \\
\text { R } \\
\text { R } \\
\text { E } \\
\text { C } \\
\text { C } \\
\text { I } \\
\text { Ó } \\
\text { N }\end{array}$} & constante & $-5,5976$ & $\star \star \star \star ~$ & $-5,6378$ & 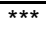 & $-5,6108$ & 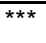 & $-5,6760$ & 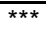 & $-5,6784$ & $\star \star \star \star ~$ & $-5,5854$ & *** \\
\hline & educación & 0,0996 & $\star \star \star \star ~$ & 0,0994 & 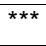 & 0,0949 & $\star \star \star \star ~$ & 0,0960 & 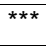 & 0,0972 & 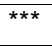 & 0,0930 & *** \\
\hline & experiencia & 0,0254 & 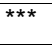 & 0,0276 & 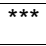 & 0,0251 & 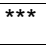 & 0,0288 & $\star \star \star *$ & 0,0270 & $\star \star \star$ & 0,0252 & 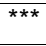 \\
\hline & experiencia2 & $-0,0003$ & 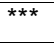 & $-0,0003$ & *** & $-0,0003$ & *** & $-0,0003$ & *** & $-0,0003$ & *** & $-0,0002$ & $\star \star \star$ \\
\hline & género & 0,0033 & & $-0,0093$ & & 0,0356 & 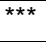 & 0,0396 & $\star * \star$ & 0,0751 & 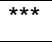 & 0,0584 & 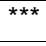 \\
\hline & jefe & 0,1351 & *** & 0,1397 & *** & 0,1353 & 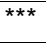 & 0,1450 & *** & 0,1356 & 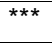 & 0,1518 & 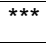 \\
\hline & & & & & & & & & & & & & \\
\hline & unipersonal & $-0,4291$ & *** & $-0,4477$ & *** & $-0,4563$ & *** & $-0,4420$ & *** & $-0,4217$ & *** & $-0,4103$ & $\star \star \star$ \\
\hline & famiempresa & $-0,3207$ & 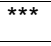 & $-0,3238$ & 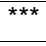 & $-0,3040$ & 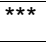 & $-0,2892$ & 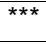 & $-0,2795$ & 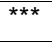 & $-0,2515$ & 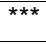 \\
\hline & microempresa & $-0,1629$ & 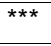 & $-0,1498$ & $\star \star \star \star$ & $-0,1727$ & 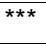 & $-0,1320$ & 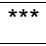 & $-0,1638$ & $\star \star \star$ & $-0,1288$ & $\star \star \star$ \\
\hline & formal pequeña & $-0,3349$ & 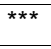 & $-0,3120$ & 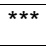 & $-0,2578$ & 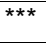 & $-0,3260$ & $\star \star \star *$ & $-0,1847$ & 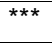 & $-0,2422$ & *** \\
\hline & $\mathrm{R} 2$ & 0,3729 & & 0,3859 & & 0,3894 & & 0,3841 & & 0,3945 & & 0,3847 & \\
\hline & Akaike & 78465,3 & & 74320,4 & & 73684,4 & & 74577,5 & & 81172,7 & & 79767,2 & \\
\hline & Schwarz & 78550,2 & & 74404,9 & & 73769,0 & & 74662,3 & & 81258,5 & & 79853,2 & \\
\hline & $\mathrm{N}$ & 36114 & & 34479 & & 35000 & & 35502 & & 39458 & & 39982 & \\
\hline & & & & & & & & & & & & & \\
\hline
\end{tabular}

Ecuaciones Mincerianas Por Tamaño 


\begin{tabular}{|c|c|c|c|c|c|c|c|c|c|c|c|c|c|}
\hline $\mathrm{C}$ & constante & $-5,4611$ & 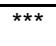 & $-5,4769$ & $\overline{k \star \star}$ & $-5,4580$ & 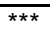 & $\begin{array}{l}-5,4788 \\
\end{array}$ & *** & $-5,6047$ & 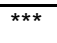 & $-5,3944$ & $\overline{\star \star \star}$ \\
\hline $\mathrm{O}$ & educación & 0,0979 & 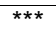 & 0,0974 & 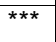 & 0,0930 & *** & 0,0935 & 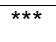 & 0,0963 & 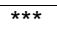 & 0,0905 & 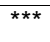 \\
\hline $\mathrm{R}$ & experiencia & 0,0208 & 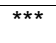 & 0,0220 & *** & 0,01986 & *** & 0,0222 & *** & 0,0245 & 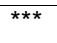 & 0,0186 & 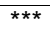 \\
\hline $\mathrm{R}$ & experiencia2 & $-0,0002$ & 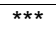 & $-0,0002$ & 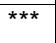 & $-0,0002$ & 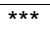 & $-0,0002$ & 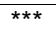 & $-0,0002$ & 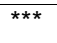 & $-0,0001$ & *** \\
\hline $\mathrm{E}$ & género & $-0,0141$ & & $-0,0294$ & *** & 0,0156 & * & 0,01238 & & 0,0639 & 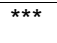 & 0,0290 & 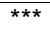 \\
\hline $\mathrm{C}$ & jefe & 0,1047 & 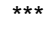 & 0,1058 & 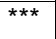 & 0,1039 & $\star \star \star \star ~$ & 0,1054 & *** & 0,1213 & *** & 0,1153 & $\star \star \star \star ~$ \\
\hline C & & & & & & & & & & & & & \\
\hline I & unipersonal & $-0,4301$ & 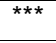 & $-0,4470$ & 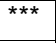 & $-0,4564$ & *** & $-0,4418$ & 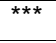 & $-0,4215$ & 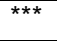 & $-0,4102$ & 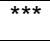 \\
\hline Ó & famiempresa & $-0,3244$ & *** & $-0,3263$ & *** & $-0,3075$ & *** & $-0,2928$ & *** & $-0,2805$ & 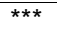 & $-0,2536$ & 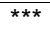 \\
\hline $\mathrm{N}$ & microempresa & $-0,1653$ & *** & $-0,1501$ & *** & $-0,1743$ & *** & $-0,1333$ & *** & $-0,1643$ & *** & $-0,1296$ & $\overline{\star \star \star \star ~}$ \\
\hline & formal pequeña & $-0,3340$ & *** & $-0,3117$ & *** & $-0,2585$ & *** & $-0,3244$ & *** & $-0,1846$ & $* \star *$ & $-0,2423$ & 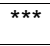 \\
\hline & lambda & $-0,0866$ & 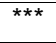 & $-0,1003$ & 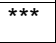 & $-0,0953$ & *** & $-0,1222$ & 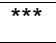 & $-0,0476$ & *** & $-0,1233$ & *** \\
\hline & $\mathrm{N}$ & 36114 & & 34479 & & 35000 & & 35502 & & 39458 & & 39982 & \\
\hline
\end{tabular}

Fuente: Procesamiento de la ECH 2001-2006 II Trimestre. Cálculos de los autores.

La comparación de los Cuadros 1 y 2 permite deducir que la inclusión de las variables de tamaño no cambia los signos de los coeficientes asociados a las variables de capital humano y de características socioeconómicas. En estas nuevas regresiones se repite el resultado anterior con respecto a la significación estadística de las variables: son siempre significativas al 1\% a excepción de la variable género. Las nuevas regresiones arrojan coeficientes negativos y significativos para las variables de tamaño. Ello implica que los trabajadores de estas empresas reciben menores remuneraciones que los trabajadores de las empresas formales grandes. Como los coeficientes son negativos y significativos de forma consistente, esto se interpreta como evidencia de segmentación laboral. El efecto tamaño sobre la remuneración laboral en 2006 se ordena de la siguiente forma: Unipersonal (-0.41), Famiempresa (-0.25), Formal Pequeña (-0.24), y Microempresa (-0.13). Este resultado es consistente con la hipótesis de segmentación de escala: a mayor tamaño, mayor remuneración laboral.

Si bien la introducción de las variables de escala no cambia los signos de los coeficientes asociados al capital humano ni su significación estadística (comparar los Cuadros 1 y 2), sí cambia su módulo, especialmente el de los coeficientes asociados con la educación, los cuales aparecen sobreestimados en las regresiones mincerianas simples (Cuadro 1). Se deduce que la no inclusión de las variables de tamaño "infla” la rentabilidad de la educación en alrededor de 1.5 puntos porcentuales. La explicación de este sesgo de escala es sencilla: dada la complementariedad entre capital físico y humano, cuando no se incluye el capital físico -o su proxy- el coeficiente asociado al capital humano refleja no sólo su propia productividad sino también la del capital físico.

\subsection{Tercer Modelo: Segundo Modelo con Efectos Locales}

Es hoy en día reconocido que en los últimos años se ha venido presentando un efecto de divergencia en términos del ingreso per cápita entre las ciudades grandes y el resto de las ciudades. Esta divergencia es más marcada cuando se compara a Bogotá con el resto de las áreas metropolitanas (Bonet y Meisel, 2007). Además, se han identificado relaciones 
estables entre informalidad y tamaño de las ciudades: a mayor tamaño de la ciudad, menor tasa de informalidad (Henao, Rojas y Parra, 1999; Ortiz y Uribe, 2000). Es válido, por tanto, formular la siguiente pregunta: ¿Es posible que la segmentación del mercado laboral colombiano a principios del siglo XXI se relacione, así sea parcialmente, con este proceso de divergencia regional?

Para investigar la pregunta formulada se introducen en nuestro modelo variables ficticias correspondientes a las áreas metropolitanas y se toma a Bogotá como referencia. El análisis se centra en las trece principales áreas metropolitanas de Colombia: Bogotá, Medellín, Cali, Barranquilla, Manizales, Pereira, Villavicencio, Pasto, Cúcuta, y Bucaramanga. El efecto local se incorpora con variables binarias que toman el valor 1 cuando representa a una ciudad y 0 en otro caso. Se tiene así la ecuación de Mincer ampliada con los efectos locales:

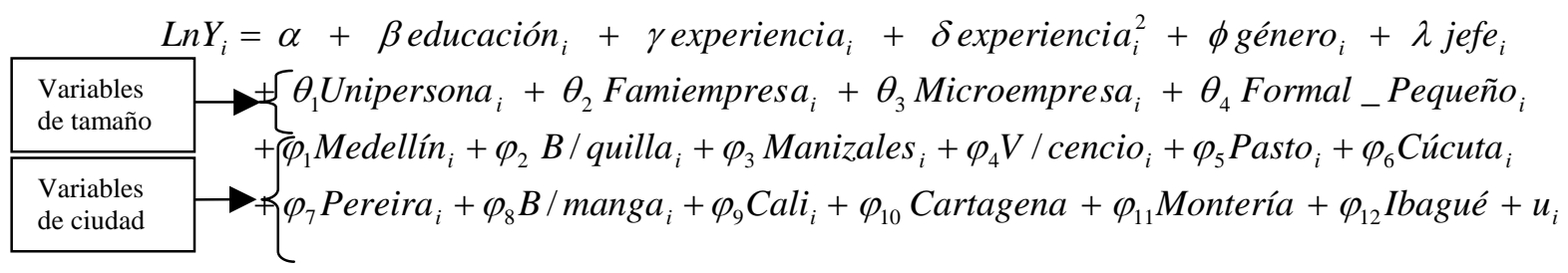

Los resultados se muestran en el Cuadro 3 sin corrección y con corrección por sesgo de selección. Se comprueba en general que con respecto a Bogotá las demás áreas metropolitanas tienen una desventaja en la generación de ingresos. Sin embargo, este fenómeno parece ser significativo y sistemático en los casos de Pasto, Barranquilla, Manizales, Montería e Ibagué.

Ser ciudad de frontera (Pasto), o ser puerto (Barranquilla), o ser ciudad intermedia (Manizales, Ibagué y Montería) son características que podrían contribuir a explicar la gran participación de actividades comerciales informales de estas ciudades (García, 2005).

Cuadro 3

Ecuaciones Mincerianas por Tamaño y Ciudad

\begin{tabular}{|c|c|c|c|c|c|c|c|c|c|c|c|c|c|}
\hline & & 2001 & & 2002 & & 2003 & & 2004 & & 2005 & & 2006 & \\
\hline Sin & constante & $-5,5658$ & 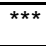 & $-5,5673$ & 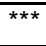 & $-5,5640$ & 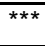 & $-5,6131$ & 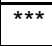 & $-5,5658$ & *** & $-5,4815$ & 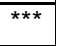 \\
\hline $\mathrm{C}$ & educación & 0,1003 & 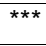 & 0,1007 & 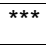 & 0,0958 & 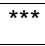 & 0,0969 & 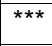 & 0,0964 & $\star \star \star$ & 0,0935 & $\star \star \star$ \\
\hline $\mathrm{O}$ & experiencia & 0,0257 & $* \star *$ & 0,0279 & 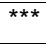 & 0,0251 & $* \star \star$ & 0,0290 & *** & 0,0265 & $\star \star \star$ & 0,0254 & 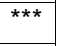 \\
\hline $\mathrm{R}$ & experiencia2 & $-0,0003$ & $* \star \star$ & $-0,0003$ & 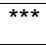 & $-0,0003$ & 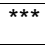 & $-0,0003$ & 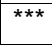 & $-0,0003$ & $\star \star \star$ & $-0,0002$ & 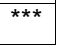 \\
\hline $\mathrm{R}$ & Género & 0,0047 & & $-0,0026$ & & 0,0396 & $\star \star \star$ & 0,0472 & 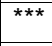 & 0,0711 & $\star \star \star$ & 0,0646 & 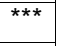 \\
\hline $\mathrm{E}$ & Jefe & 0,1310 & 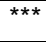 & 0,1338 & 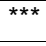 & 0,1305 & 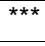 & 0,1386 & 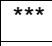 & 0,1383 & 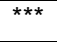 & 0,1446 & $\star \star \star$ \\
\hline $\mathrm{C}$ & & & & & & & & & & & & & \\
\hline $\mathrm{C}$ & unipersonal & $-0,4199$ & 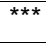 & $-0,4301$ & *** & $-0,4378$ & 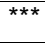 & $-0,4172$ & 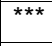 & $-0,4096$ & 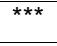 & $-0,3830$ & 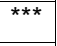 \\
\hline I & famiempresa & $-0,3102$ & 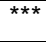 & $-0,3165$ & 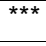 & $-0,2921$ & 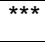 & $-0,2749$ & 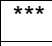 & $-0,2582$ & $\star \star \star$ & $-0,2328$ & $\star \star \star$ \\
\hline Ó & microempresa & $-0,1584$ & 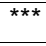 & $-0,1487$ & 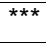 & $-0,1680$ & 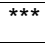 & $-0,1269$ & 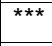 & $-0,1518$ & $\star \star \star$ & $-0,1272$ & $\star \star \star \star$ \\
\hline $\mathrm{N}$ & formal pequeña & $-0,3258$ & 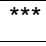 & $-0,3129$ & 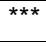 & $-0,2433$ & *** & $-0,3078$ & $\star \star \star$ & $-0,1749$ & 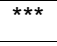 & $-0,2213$ & $\star \star \star \star$ \\
\hline & Medellín & $-0,0304$ & & $-0,0514$ & 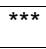 & 0,0315 & & $-0,0180$ & & $-0,0329$ & $\star \star$ & $-0,0270$ & * \\
\hline & Barranquilla & $-0,0577$ & $* \star \star$ & $-0,1595$ & 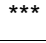 & $-0,1982$ & *** & $-0,2178$ & *** & $-0,0960$ & *** & $-0,2329$ & $\star \star \star \star ~$ \\
\hline
\end{tabular}




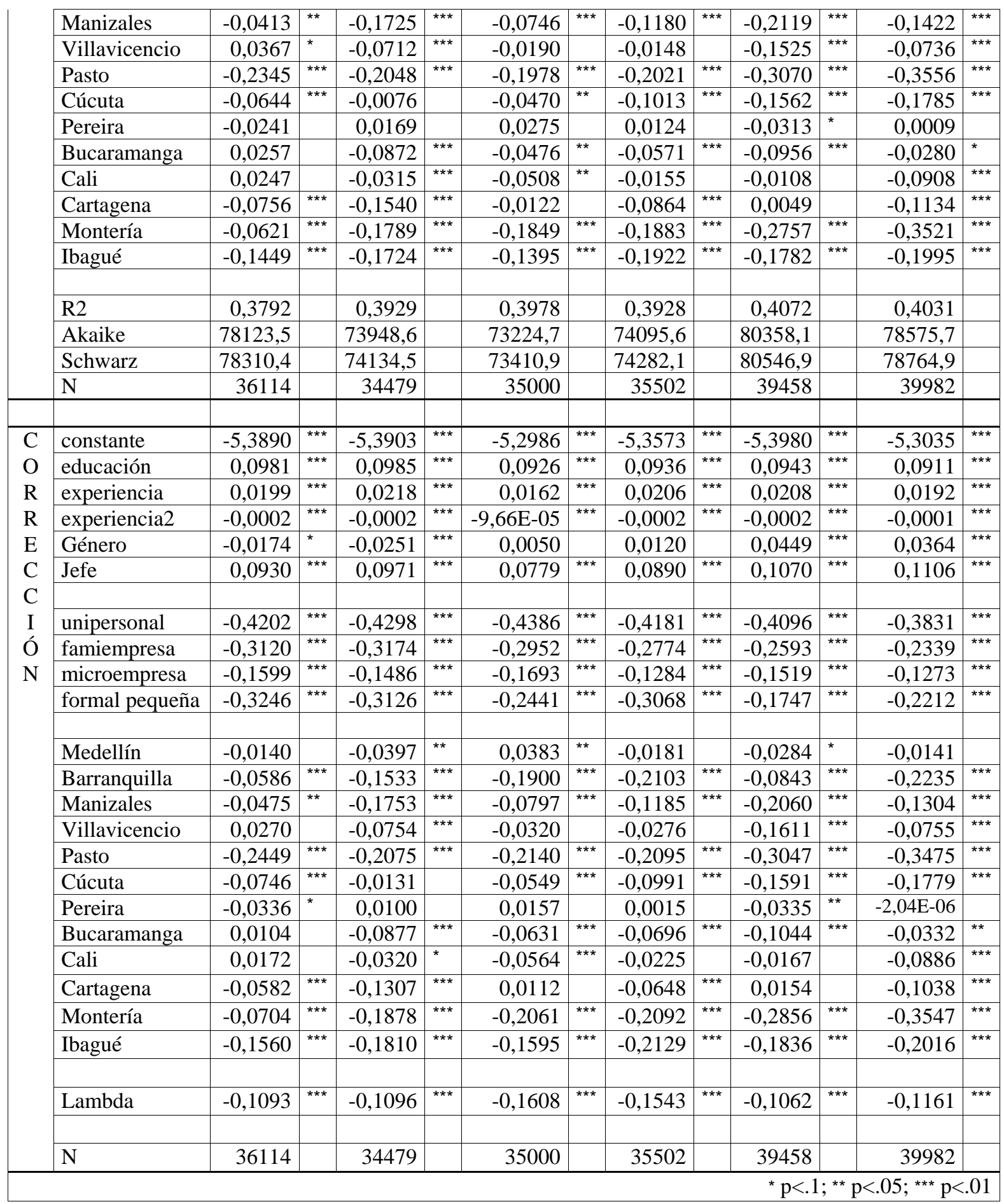

Fuente: Procesamiento de la ECH 2001-2006 II Trimestre. Cálculos de los autores.

\subsection{Cuarto Modelo: Tercer Modelo con Efectos Locales por Nivel Educativo}


Para efectos de esta investigación interesa verificar si la aparente segmentación regional del mercado laboral urbano de Colombia opera simultáneamente con la segmentación de escala. Los resultados anteriores parecen ser compatibles con esta hipótesis. Sin embargo, debe verificarse si las remuneraciones cambian con la localidad cuando se controla por el nivel educativo. Por tanto, en esta sección se modifica el modelo anterior introduciendo una variable interactiva de educación y localidad. Se tiene así la siguiente ecuación de remuneraciones:

\begin{tabular}{|c|c|}
\hline $\begin{array}{l}\text { Variables de } \\
\text { tamaño }\end{array}$ & 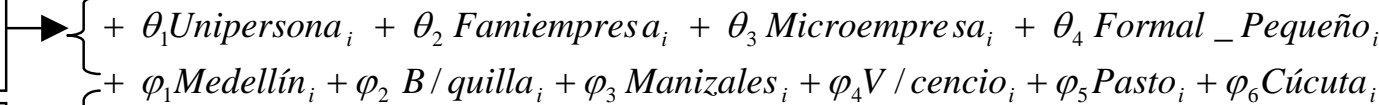 \\
\hline $\begin{array}{l}\text { Variables de } \\
\text { ciudad }\end{array}$ & $\begin{array}{l}+\varphi_{7} \text { Pereira }_{i}+\varphi_{8} B / \text { manga }_{i}+\varphi_{9} \text { Cali }_{i}+\varphi_{10} \text { Cartagena }+\varphi_{11} \text { Montería }+\varphi_{12} \text { Ibagué } \\
+\pi \text { Medellín } * \text { educación }\end{array}$ \\
\hline $\begin{array}{l}\text { Variables de } \\
\text { ciudad } x \\
\text { educación }\end{array}$ & 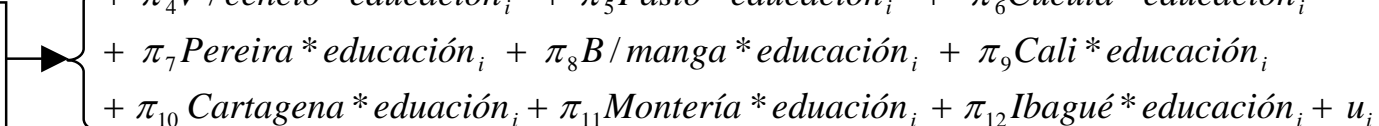 \\
\hline
\end{tabular}

Los resultados se muestran en el Cuadro 4.

Los coeficientes asociados a las variables interactivas son usualmente negativos, pero sólo son significativos de forma consistente los de Barranquilla, Villavicencio, Cúcuta, Pereira, Bucaramanga y Cartagena. Ello implica que un año adicional de educación en estas áreas metropolitanas tiene una menor remuneración que en Bogotá.

Por otra parte, los coeficientes asociados a las variables de localidad (sin interacción con la variable de educación) tienden a ser positivos y significativos en algunas áreas metropolitanas: Pereira, Medellín, Cartagena, Bucaramanga y, en menor medida, Cali y Cúcuta. Pero tienden a ser negativos y significativos en Pasto y Montería. ¿Cuáles son los atributos del primer grupo de áreas metropolitanas y las carencias del segundo que permiten entender estos resultados? Este sería un tema de investigación importante. La mayor participación del sector industrial manufacturero en la generación del PIB de Pereira, Cali, Medellín, Cartagena y Bucaramanga podría dar pistas al respecto (García, 2005).

Nótese, además, que todas las variables de tamaño siguen siendo negativas y significativas. Por tanto, la hipótesis de la coexistencia de segmentación de escala y segmentación regional no es rechazada por los datos. 


\section{Cuadro 4}

\section{Ecuaciones Mincerianas por Tamaño y Controlando la Educación por Ciudad}

\begin{tabular}{|c|c|c|c|c|c|c|c|c|c|c|c|c|c|}
\hline & & 2001 & & 2002 & & 2003 & & 2004 & & 2005 & & 2006 & \\
\hline Sin & constante & $-5,6909$ & ${ }^{* \star \star}$ & \begin{tabular}{|l|}
$-5,7079$ \\
\end{tabular} & 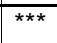 & \begin{tabular}{|c|}
$-5,7038$ \\
\end{tabular} & 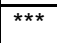 & $-5,7427$ & 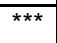 & $-5,6666$ & *** & $\begin{array}{l}-5,5520 \\
\end{array}$ & 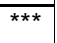 \\
\hline C & educación & 0,1125 & 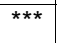 & 0,1142 & $\star \star \star \star ~$ & 0,1088 & 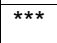 & 0,1087 & 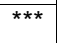 & 0,1057 & 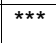 & 0,1001 & 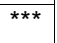 \\
\hline $\mathrm{O}$ & experiencia & 0,0256 & 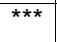 & 0,0278 & $\star \star \star \star ~$ & 0,0251 & $\star \star \star \star ~$ & 0,0289 & 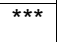 & 0,0264 & 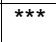 & 0,0253 & *** \\
\hline $\mathrm{R}$ & experiencia2 & $-0,0003$ & 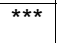 & $-0,0003$ & $\star \star \star \star ~$ & $-0,0003$ & $\star \star \star \star$ & $-0,0003$ & $\star \star \star \star ~$ & $-0,0003$ & 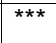 & $-0,0002$ & 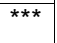 \\
\hline $\mathrm{R}$ & Género & 0,0028 & & $-0,0047$ & & 0,0368 & *** & 0,0459 & $* \star \star *$ & 0,0691 & 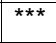 & 0,0618 & 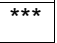 \\
\hline $\mathrm{E}$ & Jefe & 0,1309 & *** & 0,1347 & *** & 0,1328 & 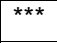 & 0,1399 & 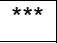 & 0,1387 & *** & 0,1452 & 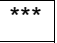 \\
\hline \multicolumn{14}{|l|}{ C } \\
\hline $\mathrm{C}$ & unipersonal & $-0,4196$ & *** & $-0,4308$ & *** & $-0,4412$ & $\star \star \star *$ & $-0,4202$ & 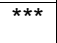 & $-0,4132$ & $\star \star \star *$ & $-0,3837$ & 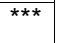 \\
\hline I & famiempresa & $-0,3093$ & 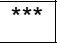 & $-0,3150$ & *** & $-0,2918$ & *** & $-0,2739$ & 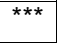 & $-0,2570$ & *** & $-0,2321$ & *** \\
\hline Ó & microempresa & $-0,1595$ & *** & $-0,1482$ & *** & $-0,1683$ & $\star \star \star$ & $-0,1276$ & 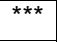 & $-0,1502$ & *** & $-0,1247$ & 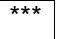 \\
\hline \multirow{29}{*}{$\mathrm{N}$} & formal pequeña & $-0,3272$ & 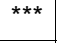 & $-0,3141$ & 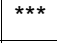 & $-0,2442$ & *** & $-0,3044$ & *** & $-0,1645$ & *** & $-0,2137$ & 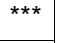 \\
\hline & Medellín & 0,0721 & & 0,1032 & ** & 0,1014 & ** & 0,0595 & & 0,0841 & 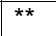 & 0,0838 & ** \\
\hline & Barranquilla & 0,1850 & $* \star *$ & 0,0450 & & 0,0017 & & $-0,0269$ & & 0,1441 & 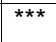 & $-0,0973$ & *** \\
\hline & Manizales & 0,0139 & & $-0,0396$ & & $-0,0029$ & & $-0,0170$ & & $-0,2793$ & 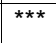 & $-0,1479$ & 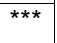 \\
\hline & Villavicencio & 0,1846 & *** & 0,0408 & & 0,1332 & *** & 0,1305 & *** & $-0,0325$ & & 0,0801 & * \\
\hline & Pasto & $-0,2066$ & 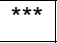 & $-0,1085$ & ** & $-0,1050$ & ** & $-0,1177$ & 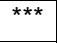 & $-0,2270$ & 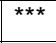 & $-0,4034$ & 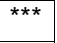 \\
\hline & Cúcuta & 0,1554 & 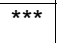 & 0,2538 & $\star \star \star \star ~$ & 0,1691 & 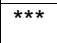 & 0,1108 & $\star \star \star$ & 0,0220 & & $-0,0249$ & \\
\hline & Pereira & 0,1403 & *** & 0,2070 & *** & 0,2062 & *** & 0,2251 & $* \star \star *$ & 0,0764 & ** & 0,1243 & *** \\
\hline & Bucaramanga & 0,2573 & 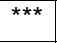 & 0,1107 & ** & 0,1485 & 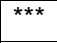 & 0,0911 & ** & 0,0409 & & 0,1388 & 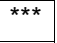 \\
\hline & Cali & 0,1185 & $\star \star \star$ & 0,0887 & * & 0,1427 & 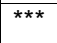 & 0,1426 & 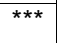 & 0,0563 & & $-0,1138$ & 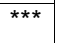 \\
\hline & Cartagena & 0,0810 & * & 0,0780 & * & 0,3206 & 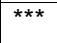 & 0,1634 & *** & 0,2973 & *** & 0,0415 & \\
\hline & Montería & 0,0391 & & $-0,1234$ & *** & $-0,1123$ & ** & $-0,0404$ & & $-0,2056$ & *** & $-0,3826$ & *** \\
\hline & Ibagué & $-0,0392$ & & $-0,0174$ & & 0,0226 & & $-0,1536$ & *** & $-0,1128$ & ** & $-0,1269$ & 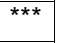 \\
\hline & $\begin{array}{l}\text { Medellín x } \\
\text { educación }\end{array}$ & $-0,0095$ & ** & $-0,0146$ & *** & $-0,0057$ & & $-0,0066$ & & $-0,0106$ & *** & $-0,0102$ & *** \\
\hline & B_quilla x educación & $-0,0244$ & *** & $-0,0197$ & $* * *$ & $-0,0189$ & *** & $-0,0176$ & *** & $-0,0223$ & *** & $-0,0126$ & *** \\
\hline & $\begin{array}{l}\text { Manizales x } \\
\text { educación }\end{array}$ & $-0,0045$ & & $-0,0123$ & *** & $-0,0058$ & & $-0,0087$ & ** & 0,0076 & ** & 0,0012 & \\
\hline & $\begin{array}{l}\text { V_cencio x } \\
\text { educación }\end{array}$ & $-0,0145$ & *** & $-0,0100$ & ** & $-0,0140$ & *** & $-0,0133$ & ${ }^{* \star *}$ & $-0,0110$ & *** & $-0,0148$ & *** \\
\hline & Pasto x educación & $-0,0016$ & & $-0,0086$ & ** & $-0,0079$ & ** & $-0,0070$ & * & $-0,0068$ & * & 0,0052 & \\
\hline & Cúcuta x educación & $-0,0239$ & *** & $-0,0285$ & 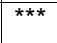 & $-0,0220$ & 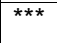 & $-0,0216$ & 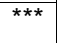 & $-0,0183$ & $* \star * \star$ & $-0,0158$ & *** \\
\hline & Pereira x educación & $-0,0167$ & 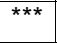 & $-0,0187$ & *** & $-0,0169$ & *** & $-0,0205$ & 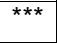 & $-0,0097$ & 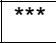 & $-0,0117$ & *** \\
\hline & $\begin{array}{l}\text { B_manga x } \\
\text { educación }\end{array}$ & $-0,0240$ & *** & $-0,0194$ & *** & $-0,0188$ & $\star \star \star *$ & $-0,0136$ & 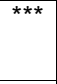 & $-0,0126$ & *** & $-0,0162$ & *** \\
\hline & Cali x educación & $-0,0087$ & ** & $-0,0110$ & ** & $-0,0185$ & $\star \star \star$ & $-0,0146$ & *** & $-0,0056$ & & 0,0032 & \\
\hline & $\begin{array}{l}\text { Cartagena x } \\
\text { educación }\end{array}$ & $-0,0154$ & 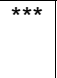 & $-0,0231$ & 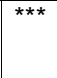 & $-0,0336$ & 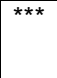 & $-0,0241$ & *** & $-0,0269$ & 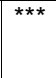 & $-0,0142$ & *** \\
\hline & $\begin{array}{l}\text { Montería x } \\
\text { educación }\end{array}$ & $-0,0093$ & ** & $-0,0041$ & & $-0,0051$ & & $-0,0136$ & *** & $-0,0057$ & & 0,0043 & \\
\hline & Ibagué x educación & $-0,0099$ & ** & $-0,0147$ & *** & $-0,0150$ & 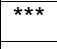 & $-0,0022$ & & $-0,0054$ & & $-0,0065$ & * \\
\hline & R2 & 0,3813 & & 0,3945 & & 0,4000 & & 0,3944 & & 0,4096 & & 0,4054 & \\
\hline & Akaike & 78026,1 & & 73878,3 & & 73119,0 & & 74027,1 & & 80227,4 & & 78449,3 & \\
\hline & Schwarz & 78314,9 & & 74165,6 & & 73406,8 & & 74315,3 & & 80519,2 & & 78741,6 & \\
\hline & $\mathrm{N}$ & 36114 & & 34479 & & 35000 & & 35502 & & 39458 & & 39982 & \\
\hline & & & & & & & & & & & & & \\
\hline
\end{tabular}




\begin{tabular}{|c|c|c|c|c|c|c|c|c|c|c|c|c|c|}
\hline $\mathrm{C}$ & constante & $-5,5206$ & 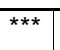 & -5,5269 & $\overline{* \star \star}$ & $-5,4389$ & 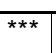 & $-5,4873$ & 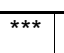 & $-5,4962$ & 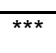 & $\begin{array}{l}-5,3758 \\
\end{array}$ & 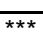 \\
\hline $\mathrm{O}$ & educación & 0,1104 & *** & 0,1120 & 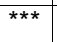 & 0,1054 & 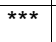 & 0,1056 & 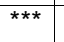 & 0,1035 & 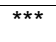 & 0,0977 & *** \\
\hline $\mathrm{R}$ & experiencia & 0,0200 & 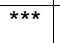 & 0,0215 & 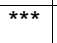 & 0,0162 & 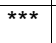 & 0,0205 & 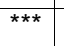 & 0,0205 & 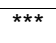 & 0,0191 & 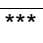 \\
\hline $\mathrm{R}$ & experiencia2 & $-0,0002$ & $\star \star \star$ & $-0,0002$ & 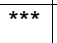 & $-9,89 \mathrm{E}-05$ & 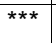 & $-0,0002$ & 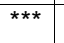 & $-0,0002$ & $\star \star \star \star$ & $-0,0001$ & 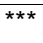 \\
\hline $\mathrm{E}$ & Género & $-0,0184$ & ** & $-0,0278$ & 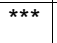 & 0,0028 & & 0,0107 & & 0,0426 & 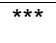 & 0,0340 & 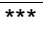 \\
\hline $\mathrm{C}$ & Jefe & 0,0943 & 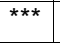 & 0,0969 & 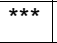 & 0,0808 & 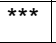 & 0,0902 & 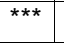 & 0,1070 & 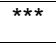 & 0,1116 & 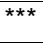 \\
\hline $\mathrm{C}$ & & & & & & & & & & & & & \\
\hline I & unipersonal & $-0,4199$ & 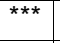 & $-0,4305$ & 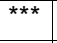 & $-0,4419$ & 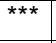 & $-0,4210$ & 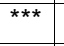 & $-0,4133$ & 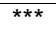 & $-0,3838$ & 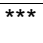 \\
\hline Ó & famiempresa & $-0,3110$ & *** & $-0,3160$ & *** & $-0,2949$ & 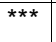 & $-0,2764$ & *** & $-0,2580$ & 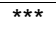 & $-0,2331$ & *** \\
\hline $\mathrm{N}$ & microempresa & $-0,1610$ & 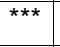 & $-0,1482$ & 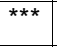 & $-0,1696$ & 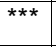 & $-0,1291$ & *** & $-0,1503$ & 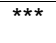 & $-0,1249$ & 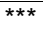 \\
\hline & formal pequeña & $-0,3260$ & 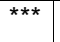 & $-0,3137$ & *** & $-0,2450$ & *** & $-0,3034$ & *** & $-0,1641$ & 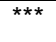 & $-0,2137$ & 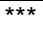 \\
\hline & Medellín & 0,0911 & 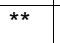 & 0,1197 & 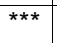 & 0,1069 & 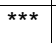 & 0,0620 & & 0,0891 & 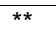 & 0,0974 & 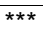 \\
\hline & Barranquilla & 0,1829 & 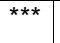 & 0,0532 & & 0,0058 & & $-0,0206$ & & 0,1573 & 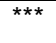 & $-0,0900$ & $\star \star \star$ \\
\hline & Manizales & 0,0078 & & $-0,0426$ & & $-0,0092$ & & $-0,0166$ & & $-0,2731$ & 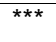 & $-0,1348$ & 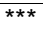 \\
\hline & Villavicencio & 0,1737 & *** & 0,0349 & & 0,1152 & *** & 0,1155 & ** & $-0,0458$ & & 0,0773 & * \\
\hline & Pasto & $-0,2161$ & *** & $-0,1103$ & *** & $-0,1236$ & 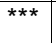 & $-0,1233$ & *** & $-0,2251$ & *** & $-0,3949$ & 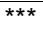 \\
\hline & Cucuta & 0,1434 & $\star \star \star$ & 0,2476 & $\star \star \star ~$ & 0,1596 & 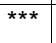 & 0,1171 & 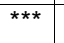 & 0,0188 & & $-0,0259$ & \\
\hline & Pereira & 0,1299 & *** & 0,2005 & *** & 0,1908 & *** & 0,2145 & *** & 0,0746 & ** & 0,1234 & *** \\
\hline & Bucaramanga & 0,2414 & 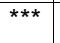 & 0,1133 & 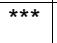 & 0,1288 & *** & 0,0794 & * & 0,0324 & & 0,1332 & 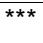 \\
\hline & Cali & 0,1134 & 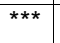 & 0,0898 & ** & 0,1373 & 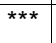 & 0,1379 & $\star \star \star$ & 0,0491 & & $-0,1112$ & 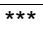 \\
\hline & Cartagena & 0,0974 & *夫 & 0,1055 & *夫 & 0,3404 & 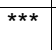 & 0,1867 & $\star \star \star$ & 0,3079 & 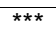 & 0,0514 & \\
\hline & Montería & 0,0302 & & $-0,1340$ & 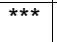 & $-0,1378$ & *** & $-0,0633$ & & $-0,2181$ & 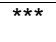 & $-0,3871$ & 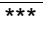 \\
\hline & Ibagué & $-0,0508$ & & $-0,0249$ & & 0,0004 & & $-0,1734$ & 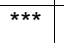 & $-0,1182$ & 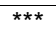 & $-0,1299$ & 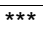 \\
\hline & $\begin{array}{l}\text { Medellín x } \\
\text { educación }\end{array}$ & $-0,0099$ & *夫 & $-0,0151$ & 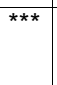 & $-0,0056$ & & $-0,0068$ & ** & $-0,0107$ & 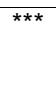 & $-0,0102$ & 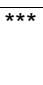 \\
\hline & B_quilla x educación & $-0,0243$ & 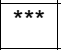 & $-0,0199$ & 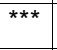 & $-0,0185$ & 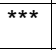 & $-0,0175$ & ${ }^{\star \star \star}$ & $-0,0224$ & 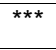 & $-0,0124$ & 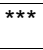 \\
\hline & $\begin{array}{l}\text { Manizales x } \\
\text { educación }\end{array}$ & $-0,0045$ & & $-0,0123$ & 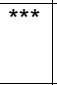 & $-0,0057$ & & $-0,0088$ & ** & 0,0076 & ** & 0,0011 & \\
\hline & $\begin{array}{l}\text { V_cencio x } \\
\text { educación }\end{array}$ & $-0,0143$ & *** & $-0,0099$ & ** & $-0,0135$ & *** & $-0,0130$ & $* * *$ & $-0,0105$ & 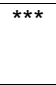 & $-0,0147$ & 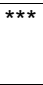 \\
\hline & Pasto x educación & $-0,0017$ & & $-0,0087$ & ** & $-0,0076$ & ** & $-0,0072$ & ** & $-0,0068$ & ** & 0,0052 & \\
\hline & Cúcuta x educación & $-0,0236$ & 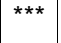 & $-0,0284$ & *** & $-0,0219$ & *** & $-0,0221$ & *** & $-0,0182$ & *** & $-0,0157$ & *** \\
\hline & Pereira x educación & $-0,0165$ & *** & $-0,0187$ & *** & $-0,0165$ & *** & $-0,0206$ & *** & $-0,0097$ & 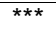 & $-0,0117$ & *** \\
\hline & $\begin{array}{l}\text { B_manga x } \\
\text { educación }\end{array}$ & $-0,0238$ & 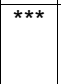 & $-0,0197$ & $\star \star \star \star$ & $-0,0184$ & 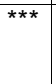 & $-0,0136$ & $\star \star \star$ & $-0,0127$ & $\star \star \star \star ~$ & $-0,0161$ & 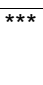 \\
\hline & Cali x educación & $-0,0089$ & ** & $-0,0112$ & *** & $-0,0186$ & *** & $-0,0148$ & *** & $-0,0054$ & * & 0,0032 & \\
\hline & $\begin{array}{l}\text { Cartagena x } \\
\text { educación }\end{array}$ & $-0,0154$ & 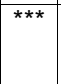 & $-0,0235$ & $\star \star \star \star$ & $-0,0333$ & 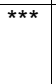 & $-0,0243$ & $\star \star \star$ & $-0,0269$ & $\star \star \star \star$ & $-0,0142$ & 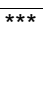 \\
\hline & $\begin{array}{l}\text { Montería x } \\
\text { educación }\end{array}$ & $-0,0092$ & ** & $-0,0039$ & & $-0,0047$ & & $-0,0133$ & *** & $-0,0054$ & * & 0,0044 & \\
\hline & Ibagué x educación & $-0,0098$ & 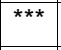 & $-0,0148$ & 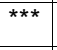 & $-0,0147$ & *** & $-0,0023$ & & $-0,0054$ & & $-0,0064$ & ** \\
\hline & Lambda & $-0,1052$ & $\star \star \star *$ & $-0,1126$ & 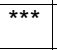 & $-0,1590$ & $\star \star \star *$ & $-0,1545$ & 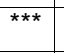 & $-0,1077$ & 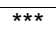 & $-0,1148$ & 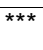 \\
\hline & $\mathrm{N}$ & 36114 & & 34479 & & 35000 & & 35502 & & 39458 & & 39982 & \\
\hline & & & & & & & & & & ${ }^{*} \mathrm{p}<.1$ & & $05 ; * \star * \mathrm{p}$ & \\
\hline
\end{tabular}

Fuente: Procesamiento de la ECH 2001-2006 II Trimestre. Cálculos de los autores. 


\subsection{Quinto Modelo: Tercer Modelo con Variables Spline de Educación}

En las anteriores estimaciones se encuentra que incluso en el sector formal de la economía existe segmentación por tamaño: los profesionales y/o técnicos independientes (que se agrupan en el sector formal pequeño) reciben de forma significativa menores remuneraciones marginales que los trabajadores de características similares en el sector formal grande (trabajadores en empresas de más de 10 trabajadores). En un trabajo anterior que utilizó la información de la década de los noventa (Uribe, Ortiz y García, 2007), se encontró que existía segmentación entre los sectores formal e informal, pero no dentro del sector formal. Este nuevo fenómeno podría deberse a dos factores: una disminución de la movilidad laboral entre empresas formales pequeñas y grandes -ampliación de la segmentación laboral-, o una disminución de la rentabilidad de la educación postsecundaria. Para contrastar esta hipótesis se corre la misma regresión minceriana con variables de tamaño y variables spline del nivel educativo. Se definen así las siguientes variables falsas (Castellar y Uribe, 2003; Greene, 2003):

$$
\begin{aligned}
& D 1_{i}\left\{\begin{array}{l}
1 \text { si educación } n_{i}>5 \text { Secundaria y Universitaria } \\
0 \text { si educación } n_{i} \leq 5
\end{array}\right. \\
& D 2_{i}\left\{\begin{array}{l}
1 \text { si educación } n_{i}>11 \text { Universitaria } \\
0 \text { si educación }{ }_{i} \leq 11 \text { Primaria y Secundaria }
\end{array}\right.
\end{aligned}
$$

El modelo que garantiza continuidad de los niveles es:

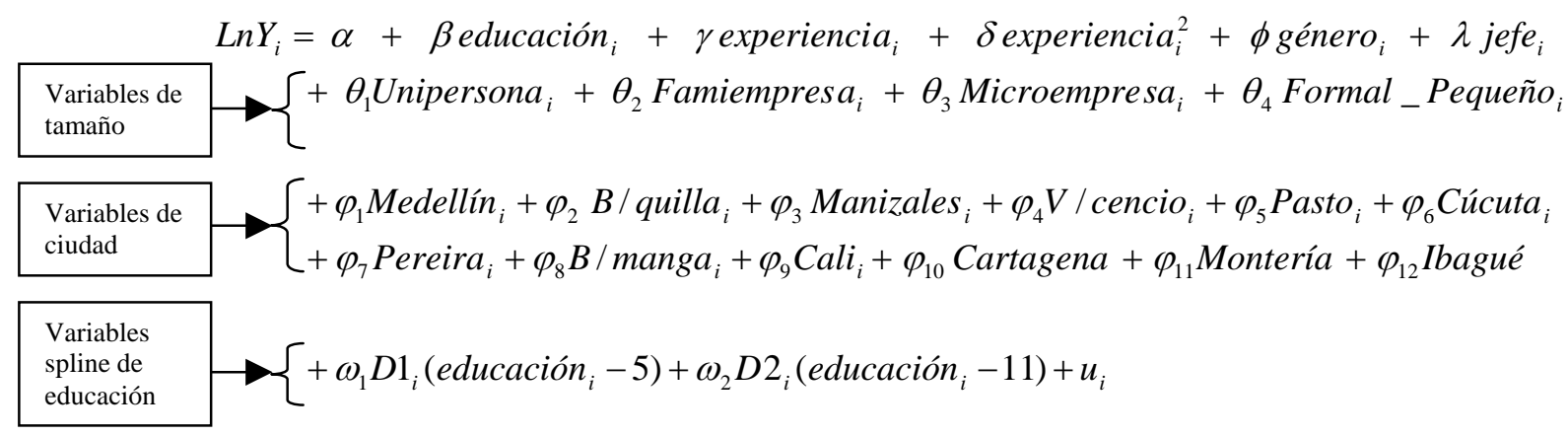

Con $D 1_{i}\left(\right.$ educación $\left._{i}-5\right)=$ spline secundaria ${ }_{i}$

$D 2_{i}\left(\right.$ educación $\left._{i}-11\right)=$ spline universita ria $_{i}$

$\beta$ : Tasa de retorno de la educación primaria

$\omega_{1}$ : Cambio en $\beta$ por educación secundaria

$\omega_{2}$ : Cambio en $\beta$ por educación universitaria

Las tasas de retorno de la educación secundaria y universitaria son:

$\beta+\omega_{1}$ : Tasa de retorno de la educación secundaria

$\beta+\omega_{2}$ : Tasa de retorno de la educación universitaria. 
Los resultados se muestran en el Cuadro 5.

Cuadro 5

Ecuaciones Mincerianas con Spline para Educación

\begin{tabular}{|c|c|c|c|c|c|c|c|c|c|c|c|c|c|}
\hline & & 2001 & & 2002 & & 2003 & & 2004 & & 2005 & & 2006 & \\
\hline \multirow{29}{*}{$\begin{array}{c}\text { Sin } \\
\mathrm{C} \\
\mathrm{O} \\
\mathrm{R} \\
\mathrm{R} \\
\mathrm{E} \\
\mathrm{C} \\
\mathrm{C} \\
\mathrm{I} \\
\mathrm{O} \\
\mathrm{N}\end{array}$} & constante & $-5,3060$ & 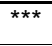 & $-5,2823$ & 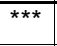 & $-5,3194$ & 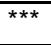 & $-5,3574$ & $\star \star \star *$ & $-5,3063$ & $* * *$ & $-5,2480$ & *** \\
\hline & educación & 0,0603 & 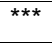 & 0,0531 & 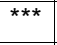 & 0,0596 & $\star \star \star$ & 0,0591 & 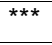 & 0,0590 & $\star \star \star$ & 0,0607 & 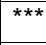 \\
\hline & experiencia & 0,0263 & $\star \star \star$ & 0,0286 & 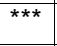 & 0,0254 & $\star \star \star$ & 0,0297 & $\star \star \star$ & 0,0271 & $* \star *$ & 0,0264 & *** \\
\hline & experiencia2 & $-0,0003$ & $\star \star \star$ & $-0,0003$ & $\star \star \star *$ & $-0,0003$ & 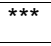 & $-0,0004$ & 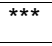 & $-0,0003$ & $* \star \star$ & $-0,0003$ & 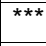 \\
\hline & Género & 0,0113 & & 0,0044 & & 0,0445 & 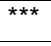 & 0,0545 & 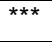 & 0,0788 & 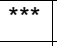 & 0,0717 & 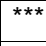 \\
\hline & Jefe & 0,1249 & $\star \star \star * *$ & 0,1300 & 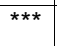 & 0,1239 & $\star \star \star$ & 0,1323 & $\star \star \star$ & 0,1311 & $\star \star \star$ & 0,1368 & 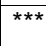 \\
\hline & & & & & & & & & & & & & \\
\hline & unipersonal & $-0,4079$ & $\star \star \star$ & $-0,4128$ & 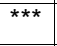 & $-0,4218$ & 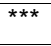 & $-0,4013$ & 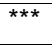 & $-0,3904$ & $* * *$ & $-0,3664$ & *** \\
\hline & famiempresa & $-0,2929$ & 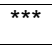 & $-0,2967$ & $\star \star \star$ & $-0,2753$ & $\star \star \star *$ & $-0,2531$ & $\star \star \star$ & $-0,2379$ & *** & $-0,2164$ & *** \\
\hline & microempresa & $-0,1405$ & $\star \star \star$ & $-0,1311$ & $\star \star \star$ & $-0,1535$ & $\star \star \star$ & $-0,1140$ & $\star \star \star$ & $-0,1324$ & $\star \star \star$ & $-0,1111$ & *** \\
\hline & formal pequeña & $-0,4772$ & 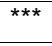 & $-0,4563$ & *** & $-0,3730$ & *** & $-0,4346$ & 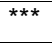 & $-0,3022$ & *** & $-0,3352$ & 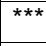 \\
\hline & Medellín & $-0,0284$ & & $-0,0467$ & ** & 0,0344 & * & $-0,0194$ & & $-0,0235$ & & $-0,0252$ & * \\
\hline & Barranquilla & $-0,0561$ & 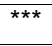 & $-0,1495$ & $\star * *$ & $-0,1915$ & 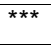 & $-0,2111$ & 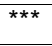 & $-0,0837$ & $* \star \star$ & $-0,2231$ & *** \\
\hline & Manizales & $-0,0352$ & * & $-0,1644$ & $\star \star \star$ & $-0,0693$ & 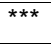 & $-0,1033$ & *** & $-0,2004$ & $\star \star \star$ & $-0,1301$ & 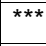 \\
\hline & Villavicencio & 0,0373 & * & $-0,0634$ & 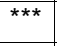 & $-0,0178$ & & $-0,0079$ & & $-0,1446$ & *** & $-0,0586$ & 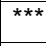 \\
\hline & Pasto & $-0,2580$ & $\star \star \star$ & $-0,2257$ & $\star \star \star$ & $-0,2058$ & $* * *$ & $-0,2265$ & *** & $-0,3261$ & $\star \star \star$ & $-0,3675$ & 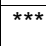 \\
\hline & Cúcuta & $-0,0829$ & $\star * \star *$ & $-0,0272$ & & $-0,0605$ & 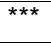 & $-0,1196$ & *** & $-0,1699$ & $* \star *$ & $-0,1901$ & 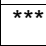 \\
\hline & Pereira & $-0,0253$ & & 0,0197 & & 0,0285 & & 0,0055 & & $-0,0320$ & ** & 0,0019 & \\
\hline & Bucaramanga & 0,0134 & & $-0,0889$ & $\star \star \star *$ & $-0,0486$ & ** & $-0,0631$ & *** & $-0,1034$ & *** & $-0,0246$ & \\
\hline & Cali & 0,0223 & & $-0,0296$ & & $-0,0444$ & ** & $-0,0176$ & & $-0,0045$ & & $-0,0866$ & 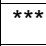 \\
\hline & Cartagena & $-0,0674$ & $\star \star \star$ & $-0,1420$ & $\star \star \star$ & $-0,0038$ & & $-0,0858$ & 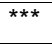 & 0,0156 & & $-0,1134$ & *** \\
\hline & Montería & $-0,0729$ & $\star \star \star$ & $-0,1874$ & 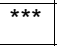 & $-0,1861$ & 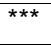 & $-0,1940$ & 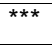 & $-0,2799$ & 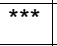 & $-0,3561$ & $\star \star \star$ \\
\hline & Ibagué & $-0,1476$ & 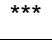 & $-0,1721$ & $\star \star \star$ & $-0,1371$ & $\star \star \star ~$ & $-0,1998$ & *** & $-0,1706$ & 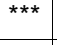 & $-0,1940$ & 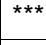 \\
\hline & Spline secundaria & 0,0094 & * & 0,0192 & $\star \star \star *$ & 0,0052 & & 0,0066 & & 0,0045 & & 0,0011 & \\
\hline & Spline universitaria & 0,0718 & 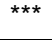 & 0,0671 & 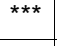 & 0,0674 & *** & 0,0677 & $\star \star \star *$ & 0,0691 & $\star \star \star *$ & 0,0651 & 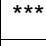 \\
\hline & R2 & 0,3942 & & 0,4076 & & 0,4116 & & 0,4069 & & 0,4221 & & 0,4170 & \\
\hline & Akaike & 77247,3 & & 73106,7 & & 72414,0 & & 73267,2 & & 79358,6 & & 77635,4 & \\
\hline & Schwarz & 77451,2 & & 73309,4 & & 72617,1 & & 73470,7 & & 79564,6 & & 77841,7 & \\
\hline & $\mathrm{N}$ & 36114 & & 34479 & & 35000 & & 35502 & & 39458 & & 39982 & \\
\hline $\mathrm{C}$ & constante & $-5,1285$ & *** & $-5,1050$ & 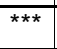 & $-5,0531$ & 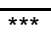 & $-5,0912$ & *** & $-5,1177$ & $* * *$ & $-5,0723$ & *** \\
\hline $\mathrm{O}$ & educación & 0,0579 & $\star \star \star$ & 0,0508 & $\star \star \star *$ & 0,0564 & $\star \star \star$ & 0,0552 & $* \star \star$ & 0,0561 & 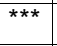 & 0,0577 & *** \\
\hline $\mathrm{R}$ & experiencia & 0,0205 & 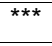 & 0,0225 & $\star \star \star$ & 0,0165 & $\star \star \star$ & 0,0210 & 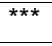 & 0,0207 & $\star \star \star$ & 0,0204 & *** \\
\hline $\mathrm{R}$ & experiencia2 & $-0,0002$ & $\star \star \star$ & $-0,0002$ & $\star \star \star$ & $-0,0001$ & $\star \star \star$ & $-0,0002$ & $\star \star \star$ & $-0,0002$ & $\star \star \star$ & $-0,0002$ & $\star \star \star$ \\
\hline $\mathrm{E}$ & Género & $-0,0108$ & & $-0,0181$ & * & 0,0099 & & 0,0182 & ** & 0,0498 & $\star \star \star$ & 0,0442 & 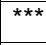 \\
\hline $\mathrm{C}$ & Jefe & 0,0870 & $\star \star \star$ & 0,0932 & 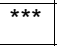 & 0,0712 & 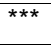 & 0,0810 & $\star \star \star$ & 0,0964 & 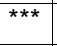 & 0,1036 & $\star \star \star \star ~$ \\
\hline $\mathrm{C}$ & & & & & & & & & & & & & \\
\hline I & unipersonal & $-0,4082$ & 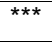 & $-0,4125$ & $\star \star \star$ & $-0,4226$ & 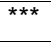 & $-0,4021$ & 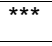 & $-0,3903$ & 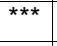 & $-0,3665$ & *** \\
\hline Ó & famiempresa & $-0,2947$ & $\star \star \star$ & $-0,2976$ & 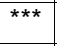 & $-0,2784$ & $\star \star \star$ & $-0,2556$ & 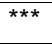 & $-0,2389$ & $\star \star \star$ & $-0,2174$ & *** \\
\hline $\mathrm{N}$ & microempresa & $-0,1420$ & $\star \star \star *$ & $-0,1311$ & $* * *$ & $-0,1548$ & *** & $-0,1154$ & $\star \star \star *$ & $-0,1324$ & $\star \star \star *$ & $-0,1113$ & *** \\
\hline & formal pequeña & $-0,4760$ & 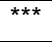 & $-0,4560$ & $\star \star \star *$ & $-0,3738$ & 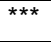 & $-0,4338$ & 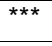 & $-0,3024$ & *** & $-0,3349$ & 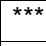 \\
\hline & Medellín & $-0,0121$ & & $-0,0349$ & * & 0,0412 & $\star \star$ & $-0,0194$ & & $-0,0184$ & & $-0,0126$ & \\
\hline & Barranquilla & $-0,0571$ & 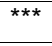 & $-0,1433$ & $\star \star \star$ & $-0,1833$ & $\star \star \star ~$ & $-0,2033$ & 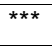 & $-0,0707$ & 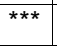 & $-0,2139$ & *** \\
\hline
\end{tabular}




\begin{tabular}{|c|c|c|c|c|c|c|c|c|c|c|c|c|}
\hline Manizales & $-0,0414$ & ** & $-0,1671$ & $* \star \star$ & $-0,0745$ & *** & $-0,1038$ & 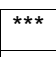 & $-0,1938$ & 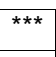 & $-0,1186$ & *** \\
\hline Villavicencio & 0,0276 & & $-0,0676$ & *** & $-0,0309$ & & $-0,0211$ & & $-0,1542$ & *** & $-0,0606$ & 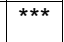 \\
\hline Pasto & $-0,2684$ & 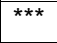 & $-0,2284$ & 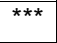 & $-0,2221$ & 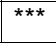 & $-0,2342$ & *** & $-0,3237$ & 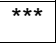 & $-0,3596$ & *** \\
\hline Cúcuta & $-0,0932$ & 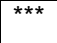 & $-0,0327$ & * & $-0,0684$ & 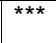 & $-0,1173$ & 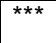 & $-0,1731$ & 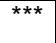 & $-0,1896$ & 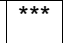 \\
\hline Pereira & $-0,0348$ & * & 0,0128 & & 0,0167 & & $-0,0058$ & & $-0,0344$ & ** & 0,0011 & \\
\hline Bucaramanga & $-0,0019$ & & $-0,0894$ & $\star \star \star \star ~$ & $-0,0641$ & 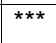 & $-0,0760$ & $\star \star \star \star ~$ & \begin{tabular}{|l|}
$-0,1133$ \\
\end{tabular} & 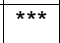 & $-0,0296$ & * \\
\hline Cali & 0,0148 & & $-0,0302$ & & $-0,0500$ & ** & $-0,0249$ & & $-0,0111$ & & $-0,0845$ & 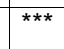 \\
\hline Cartagena & $-0,0500$ & ** & $-0,1186$ & 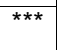 & 0,0196 & & $-0,0635$ & *** & 0,0274 & & $-0,1041$ & *** \\
\hline Montería & $-0,0813$ & *** & $-0,1963$ & *** & $-0,2074$ & $* \star *$ & $-0,2157$ & *** & $-0,2910$ & $* * *$ & $-0,3588$ & *** \\
\hline Ibagué & $-0,1587$ & 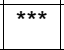 & $-0,1807$ & 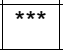 & $-0,1571$ & 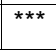 & $-0,2211$ & 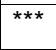 & $-0,1766$ & *** & $-0,1960$ & 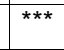 \\
\hline Spline secundaria & 0098 & ** & 0,0193 & $\star \star \star$ & 0,0053 & & 0,0072 & & 0,0050 & & 0,0020 & \\
\hline Spline universitaria & 0,0717 & 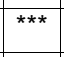 & 0,0671 & $\star \star \star *$ & 0,0674 & 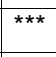 & 0,0678 & *** & 0,0693 & 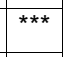 & 0,0648 & *** \\
\hline Lambda & $-0,1093$ & 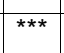 & $-0,1097$ & 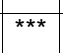 & $-0,1612$ & 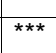 & $-0,1594$ & 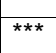 & $-0,1180$ & *** & $-0,1134$ & 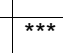 \\
\hline $\mathrm{N}$ & 36114 & & 34479 & & 35000 & & 35502 & & 39458 & & 39982 & \\
\hline
\end{tabular}

Fuente: Procesamiento de la ECH 2001-2006 II Trimestre. Cálculos de los autores.

Los resultados revelan que las variables de tamaño siguen siendo negativas y significativas. También muestran que la educación primaria (variable Educación) y la educación profesional (variable Spline Universitaria) tienen efectos positivos y significativos sobre la rentabilidad de la educación. El mayor aporte marginal a la rentabilidad de la educación le corresponde a la educación superior. Por su parte, la educación secundaria (variable Spline Secundaria) no le aporta prácticamente nada a esa rentabilidad: su coeficiente estimado es positivo pero generalmente no es significativo. Así, pues, el modelo permite postular que no es rentable abandonar los estudios en la secundaria. Además, se puede concluir que la formación universitaria no se ha desvalorizado, sino que la menor remuneración de los profesionales y técnicos independientes se debe a fuertes barreras a la movilidad por efecto de tamaño.

Una comparación de los cinco modelos estimados permite concluir, de acuerdo con los indicadores de Akaike y Schwarz, que el quinto modelo presenta la mejor bondad de ajuste.

\section{Conclusiones}

En este trabajo se ha considerado un modelo reducido de determinación de las remuneraciones laborales que incorpora variables relevantes en forma aditiva. En el primer modelo, siguiendo la tradición de las ecuaciones mincerianas, sólo se incluyen variables de la oferta laboral (capital humano y características socioeconómicas del individuo); en el segundo modelo se incluyen adicionalmente variables de la demanda laboral (efecto del tamaño o de escala de las firmas); en el tercer modelo se incorporan variables regionales (variables ficticias por áreas metropolitanas); con base en el modelo anterior, se examina en el cuarto modelo la interacción entre las variables de educación y las variables regionales; finalmente, en el quinto modelo se considera el tercer modelo junto con los efectos acumulativos de los niveles educativos (primaria, secundaria y superior). 
En general, las estimaciones son consistentes con la hipótesis de segmentación laboral por efectos de escala (intrarregional) y por regiones (interregional): la remuneración laboral es significativamente mayor en las empresas formales grandes y en Bogotá. Más aún, la consideración simultánea de estas dos dimensiones de la segmentación mejora el ajuste del modelo.

La estimación del cuarto modelo muestra que los coeficientes asociados a las variables interactivas entre educación y región son usualmente negativos. Ello implicaría que un año adicional de educación tiene una menor remuneración que en Bogotá. Este efecto, sin embargo, sólo es significativo de forma consistente en los casos de Barranquilla, Villavicencio, Cúcuta, Pereira, Bucaramanga y Cartagena.

Del modelo con variables por nivel educativo (primaria, secundaria y superior) se deduce que la rentabilidad agregada de la educación se explica primordialmente por el aporte de la educación primaria y la educación universitaria, siendo esta última más importante. De acuerdo con las estimaciones realizadas, la educación secundaria le aporta poco o nada a la rentabilidad de la educación.

La inclusión del efecto de escala (tamaño de las firmas) corrige lo que se ha denominado el sesgo de escala: la no inclusión de las variables de tamaño de las empresas induce una sobreestimación de la rentabilidad de la educación.

El análisis econométrico es consistente con la hipótesis de segmentación laboral entre los diferentes subconjuntos considerados (unipersonales, famiempresas, microempresas y sector formal pequeño) y el sector formal grande.

La existencia de segmentación regional implicaría la necesidad de utilizar políticas de desarrollo regional para compensar las desigualdades y promover el desarrollo nacional. Por otra parte, la segmentación de escala exigiría tratar de forma diferente a las empresas según su tamaño, pues mientras las empresas grandes siguen una lógica de acumulación de capital, las pequeñas siguen una lógica de subsistencia, y las empresas intermedias (fami y microempresas) están a medio camino. Disminuir los costos de transacción para las empresas pequeñas y facilitar el acceso al mercado de capitales (físico y humano) son medidas necesarias para promover el desarrollo del mercado laboral urbano en Colombia.

\section{Referencias Bibliográficas}

ALBÆK, K.; ARAI, M.; ASPLUND, R.; BARTH, E. y MADSEN E. S. (1998). "Measuring Wage Effects of Plant Size”, Labour Economics, 5, pp. 425-448.

AMARAL, P. y QUINTÍN E. (2003). “The implications of Capital- Skill Complementarity in Economies with Large Informal Sectors”, Working Paper, Federal Reserve Bank of Dallas. 
BARÓN, J. D. (2004). “¿Qué Sucedió con las Disparidades Económicas Regionales en Colombia entre 1980 y el 2000?”, en A. Meisel (editor), Macroeconomía y Regiones en Colombia, Banco de la República, Cartagena.

BID (2004). Good Jobs Wanted. Labor Markets in Latin America. Economic and Social Progress in Latin America, Washington.

BIRCHENALL, J. y MURCIA, G. (1996), “Convergencia Regional: Una Revisión del Caso Colombiano”, Desarrollo y Sociedad, No. 40, pp. 273-308, Universidad de los Andes, Bogotá.

BONET, J. y MEISEL, A. (1999). "La Convergencia Regional en Colombia: Una Visión de Largo Plazo, 1926-1995”, Coyuntura Económica, 29, 1, pp. 69-106.

BONET, J. y MEISEL, A. (2007). "Polarización del Ingreso Per Cápita Departamental en Colombia, 1975-2000”, Ensayos sobre Política Económica, No. 54, pp. 14-43, Banco de la República, Bogotá.

BOURGUIGNON, F. (1979). "Pobreza y Dualismo en el Sector Urbano de las Economías en Desarrollo: El Caso de Colombia”, Desarrollo y Sociedad, No. 1, Universidad de los Andes, Bogota.

(1983). “El Papel de la Educación en el Mercado de Trabajo Urbano en el Proceso de Desarrollo: El Caso de Colombia”, cap. 13 en Urquidi, Victor L. y Saúl Trejo Reyes, Recursos Humanos, Empleo y Desarrollo en la América Latina, Fondo de Cultura Económica, Lecturas No. 51, El Trimestre Económico, México.

BROWN, C. y MEDOFF J. L. (1989). “The Employer Size-Wage Effect”, Journal of Political Economy, 97, pp. 1027-1059.

BRUNELLO, G. y COLUSSI, A. (1998). “The Employer Size-Wage Effect: Evidence from Italy”, Labour Economics, 5, pp. 217-230.

CAIN, G. (1976). “The Challenge of Segmented Labor Market Theories to Orthodox Theory: A Survey”, Journal of Economic Literature, Vol. 15, No 4.

CASTELLAR, C. y URIBE J. I. (2003). “Capital Humano y Señalización: Evidencia para el Área Metropolitana de Cali 1988-200”, Documentos de Trabajo, No. 65, CIDSE, Universidad del Valle.

CEGA (Centro de Estudios Ganaderos y Agrícolas) (2006). Ingreso, Consumo y Ahorro en los Departamentos de Colombia 1975 a 2000: Sistema Simplificado de Cuentas Departamentales de Colombia (SSCD), Bogotá.

DANE, Cuentas Departamentales, varios años.

DANE, Encuesta Continua de Hogares, 2001-2006. 
DE SOTO, H. (1987). El Otro Sendero, la Revolución Informal, Instituto Libertad y Democracia, Lima.

DICKENS, W. T. y LANG, K. (1985). “A Test of Dual Labour Market Theory”, American Economic Review, No. 75, 4, pp. 792-805.

DOERINGER, P. y PIORE, M. (1971). “Internal Labour Markets and Manpower Analysis”, Lexington, Mass., Lexington Books. Versión española en Toharia, Luis (1983). El Mercado de trabajo: teorías y aplicaciones, pp. 341-368, Alianza Universidad Textos, Madrid.

$\mathrm{y}$ (1975). "Unemployment and the Dual Labor Markets”, The Public Interest, No. 38, 67-79. Versión española en Toharia, Luis (1983). El Mercado de trabajo: teorías y aplicaciones, pp. 307-320, Alianza Universidad Textos, Madrid.

EASTERLY, W. y LEVINE, R. (2001). "It's Not Factor Accumulation: Stylized Facts and Growth Models", World Bank Economic Review, Vol. 15, No. 2, pp. 177-220.

FEI J.C.H. y G. RANIS. (1964). Development of the Labor Surplus Economy: Theory and Policy, New Haven, Conn.: Yale University Press.

FIELDS, G. (1980). “How Segmented is the Bogotá Labor Market?”, World Bank Staff Working Paper, No. 434, October.

FIELDS, G. (2004). A Guide to Multisector Labor Market Models, Cornell University, Paper Prepared for the World Bank Labor Market Conference, Washington, November 1819.

GALVIS, L. A. (2004). "Integración Regional de los Mercados Laborales en Colombia, 1984-2000”, Macroeconomía y Regiones en Colombia, Adolfo Meisel (ed.), Colección de Economía Regional, Banco de la República, Cartagena.

GARCÍA CRUZ, G. A. (2005). "El Componente Local de la Informalidad Laboral para las Diez Principales Áreas Metropolitanas de Colombia, 1988-2000”, Desarrollo y Sociedad, No. 56, pp. 103-146, Universidad de los Andes, Bogotá.

GRACIA, O.; HERNÁNDEZ, G. y RAMIREZ, J. M. (2001). “Diferenciales Salariales y Mercados Laborales en la Industria Colombiana”, Desarrollo y Sociedad, No. 48, Universidad de los Andes, Bogotá.

GREENE, W. (2003). Econometrics Analysis. Prentice Hall, New Jersey: Fifth Edition.

GRILICHES, Z. (1969). “Capital-Skill Complementarity”, Review of Economics and Statistics, 51, pp. 465-468. 
HALL, R. E. y JONES, C. I. (1997). "Levels of Economic Activity across Countries”, American Economic Review, Vol. 87, No. 2, pp. 173-177, Papers and Proceedings of the Hundred and Fourth Annual Meeting of the American Economic Association, May.

HARRIS, J. y TODARO, M. (1970). "Migration, Unemployment, and Development: A Two Sector Analysis”, American Economic Review, 60, pp. 126-142, March.

HART, K. (1973). “Informal Income Opportunities and Urban Unemployment in Ghana”, Journal of Modern African Studies, 11, pp. 61-89.

HECKMAN, J.J. (1979). “Sample Selection Bias as a Specification Error”, Econometrica, Vol. 47, No. 1, pp. 152-161, January.

HENAO, M.; ROJAS, N. y PARRA, A. (1999). "EL Mercado Laboral Urbano y la Informalidad en Colombia: Evolución Reciente”, Revista Planeación y Desarrollo, Volumen XXX, Numero 2, Abril-Junio.

HIRSCHMAN, A. (1958). The Strategy of Economic Development, Yale University Press, Inc., New Haven.

HUGUET, A. y SÁNCHEZ, M. (2001). “Efectos Selección e Impacto del Tamaño del Empleador sobre los Salarios: El Caso Español”, Documento de Trabajo, No. 6, Departamento de Análisis Económico, Universidad de Valencia, España.

IREGUI, A.M.; MELO L.F. y RAMÍREZ M.T. (2007). "Productividad Regional y Sectorial en Colombia: Un Análisis Utilizando Datos de Panel”, Ensayos Sobre Política Económica, Vol. 25, No. 53, pp. 18-65.

LANG, K. y W.T. DICKENS, (1984). “A Test of Dual Labor Market Theory”, National Bureau of Economic Research NBER, Working Paper, 1314.

y _ (1985). “Testing Dual Labor Market Theory: A Reconsideration of the Evidence”, National Bureau of Economic Research NBER, Working Paper Series, 1670.

y _ (1987). "Neoclassical and Sociological Perspectives on Segmented Labor Markets”, National Bureau of Economic Research NBER, Working Paper Series, 2127.

LEIBOVICH J.; NIGRINIS M. y RAMOS M. A. (2006). “Caracterización del mercado laboral rural en Colombia”, Borradores de Economía, No. 408, Banco de la República, Bogotá.

LEWIS, A. (1954). "Economic Development with Unlimited Supplies of Labor", The Manchester School of Economic and Social Studies, 22, pp. 139-191, May. 
LOAYZA, N. (1997). “The Economics of the Informal Sector. A Simple Model and Empirical Evidence from Latin America”, Policy Research Working Paper, No. 1727, February.

MADDISON, A. (1994). "Explaining the Economic Performance of Nations, 1820-1989”, en W.J.

MAGNAC, T. (1991), “Segmented or Competitive Labor Markets”, Econometrica, Vol. 59, No. 1, pp. 165- 187, January.

MAIN, B. G. M. y REILLY, B. (1993). “The Employer Size-Wage Gap: Evidence for Britain”, Económica, 60, pp. 125-142.

MALONEY, W. (1998a). “The Structure of Labor Markets in Developing Countries, Time Series Evidence on Competing Views”, World Bank, Policy Research Working Paper, 1940.

(1998b). “Are Labor Markets in Development Countries Dualistic?”, World Bank, Policy Research Working Paper, 1941.

(1999). "Does Informality Imply Segmentation in Urban Labor Markets? Evidence from Sectoral Transitions in Mexico”. World Bank Economic Review, Vol. 13, No. 2, pp. 275-302.

Paper, 2965.

(2003). “Informality Revisited”, World Bank, Policy Research Working

McCONNELL, C.R. y BRUE, S.L. (1997). Economía Laboral, traducido de Contemporary Labor Economics, McGraw-Hill, 1995.

MEISEL, A. (1993). “Polarización o Convergencia? A Propósito de Cárdenas, Pontón y Trujillo”, Coyuntura Económica, 23, 2, pp. 153-160.

MORA, J. y SALAZAR, B. (1994). “Fábula y Trama en el Relato de la Convergencia”, Boletín Socioeconómico, 27, CIDSE, Universidad del Valle.

MELLOW, W. (1982). "Employer Size and Wages”, Review of Economics and Statistics, 64, pp. 495-501.

OIT (1972). Employment, Incomes, and Equality: A Strategy for Increasing Productive Employment in Kenya, Ginebra.

OOSTERBEK, H. y VAN PRAAG, M. (1995). "Firm-Size Wage Differentials in the Netherlands”, Small Business Economics, 7, pp. 173-182. 
OROVAL, E. y ESCARDÍBUL, O. (1998). Economía de la Educación, Ediciones Encuentro, Madrid.

ORTIZ, C. H. y URIBE, J. I. (2000). “Mercado Laboral en el Área Metropolitana de Cali”, capítulo del libro La Economía del Valle del Cauca, Observatorio Económico del Valle del Cauca, No. 2, Primer trimestre, pp. 71-83.

Internacional”, Documento de Trabajo, No. 75, CIDSE, Universidad del Valle.

ORTIZ, C. H.; URIBE, J. I. y GARCÍA, G. A. (2007). "Segmentación de Escala y Segmentación Regional en el Mercado Laboral Urbano de Colombia 2000-2005”, en El Desarrollo: Perspectivas y Dimensiones: aportes interdisciplinarios, Carlos Zorro Sánchez (Comp.), 2007, CIDER, Universidad de los Andes, Bogotá, pp. 227-258.

PAGÉS, C. y STAMPINI, M. (2007). "No Education, No Good Jobs? Evidence on the Relationship between Education and Labor Market Segmentation”, IZA DP No. 3187, November.

PERLBACH, I. y CALDERÓN, M. (1998). "Estimación del Sesgo de Selección para el Mercado Laboral de Mendoza”, en www.aaep.org.ar/espa/anales/, referenciado como: pdf_98/perlbach-de-maradona_calderon.pdf

PERRY, G.; ARIAS, O.; MALONEY, W.; LÓPEZ, J. H. y SERVÉN, L. (2006). Poverty Reduction and Growth: Virtuous and Vicious Circles, World Bank Latin American and Caribbean Studies, Washington.

PERRY, G.; MALONEY, W.; ARIAS, O.; FAJNZYLBER, P.; MASON, A. y SAAVEDRA-CHANDUVI, J. (2007). Informality: Exit and Exclusion. The World Bank.

PIORE, M. (1975). “Notes for a Theory of Labor Market Stratification” en Richard Edwards, Michael Reich y David Gordon, eds., Labor Market Segmentation, Lexington, Mass, pp. 125-149. Existe traducción al español en Luis Toharia (compilador), El Mercado de Trabajo: Teorías y Aplicaciones, Lectura 8, Alianza Universidad, Textos, Madrid, 1983.

. (1980). "The Technological Foundations of Dualism and Discontinuity”, cap. 3 de Dualism and Discontinuity in Industrial Societies, Cambridge University Press, pp. 5581. Existe traducción al español en Luis Toharia (compilador), El Mercado de Trabajo: Teorías y Aplicaciones, Lectura 10, Alianza Universidad Textos, Madrid, 1983.

. (1983). "Notas para una Teoría de la Estratificación del Mercado de Trabajo”, en Luis Toharia (compilador), El Mercado de Trabajo: Teorías y Aplicaciones, Alianza Universidad Textos, Madrid. 
PRITCHETT, L. (1997). “Divergence, Big Time”, Journal of Economic Perspectives, Vol. 11, No. 3, pp. 3-17.

RANIS G. y FEI J.C.H. (1961). “A Theory of Economic Development,” American Economic Review, 51, pp. 533-565.

REBITZER, J. y ROBINSON, M. D. (1991). “Employer Size and Dual Labor Markets”, Review of Economics and Statistics, 73, pp. 710-715.

REILLY, K. T. (1995). "Human Capital and Information: The Employer Size-Wage Effect”, Journal of Human Resources, 30, pp. 1-18.

ROCHA, R. y VIVAS, A. (1998). "Crecimiento Regional en Colombia: ¿Persiste la Desigualdad?”, Revista de Economía del Rosario, Vol. 1, No. 1, pp. 67-108.

ROMERO, J. (2006). "Diferencias Sociales y Regionales en el Ingreso Laboral de las Principales Ciudades Colombianas, 2001-2004”, Documentos de Trabajo Sobre Economía Regional, No 67, Banco de la República, Cartagena. Enero.

ROSENZWEIG, M. (1988). “Labor Markets in Low Income Countries”, en Hollis Chenery y T.N. Srinivasan, eds., Handbook of Development Economics, Vol. 1, (Amsterdam: North Holland).

SAINT-PAUL, G. (1996). Dual Labor Markets. A Macroeconomic Perspective, The MIT Press, Cambridge, Massachussets.

SOLIMANO, A. (1988). "Enfoques Alternativos sobre el Mercado de Trabajo: Una Evolución Teórica”, Revista de Análisis Económico, Vol. 3, No. 2, November.

STIGLITZ, J. E. (1974). “Alternative Theories of Wage Determination and Unemployment in LDCs: the Labour Turnover Model”, Quarterly Journal of Economics, Vol. 98, pp. 194227.

(1982). "The Structure of Labor Markets and Shadow Prices in LDCs,” in Richard H. Sabot, ed., Migration and the Labor Market in Developing Countries. (Boulder, CO: Westview).

TAUBMAN, P. y WACHTER, M. (1986). “Segmented Labor Markets”, en Handbook of Labor Economics, T. II, ed. Elsevier Science Publishers. Traducido al español como "Mercados de Trabajo Segmentados", en ASHENFELTER y LAYARD, Manual de Economía del Trabajo, Tomo II, pp. 1519-1564, Ministerio del Trabajo y Seguridad Social, Madrid. 
URIBE, J. I.; ORTIZ, C. H. y GARCÍA, G. A. (2007). "La Segmentación del Mercado Laboral Colombiano en la Década de los Noventa”, Revista de Economía Institucional, Vol. 9, No. 16, pp. 189-221, primer semestre, Universidad Externado de Colombia, Bogotá.

URIBE, J. I. y ORTIZ, C. H. (2006). Informalidad Laboral en Colombia 1988-2000: Evolución, Teorías y Modelos. Programa Editorial Universidad del Valle, Cali. 